\title{
On the Chaotic Behavior of a Compressed Beam
}

\author{
Flaviano Battelli, Michal Fečkan, and Matteo Franca
}

Communicated by Michal Fečkan, received January 6, 2007.

\begin{abstract}
We study a PDE modelling a compressed beam with small friction and subjected to a periodic forcing of small amplitude. We assume that the load of the beam is resonant to the $i$-th eigenvalue of the associated unperturbed problem and prove that, when both forcing and damping are sufficiently small the equation exhibits chaotic behaviour.
\end{abstract}

\section{Contents}

1. Introduction 55

2. Formulation of weak solutions $\quad 56$

3. Chaotic Solutions $\quad 57$

References $\quad 85$

\section{Introduction}

This paper is devoted to the study of a system modelling a compressed beam with friction subjected to a small periodic forcing. In particular we want to individuate the existence of chaotic patterns. The model is described by the following PDE

$$
\begin{gathered}
u_{t t}+u_{x x x x}+\gamma u_{x x}-\kappa u_{x x} f\left(\int_{0}^{\pi} u_{x}^{2}(\xi, t) d \xi\right)=\epsilon\left(\nu h(x, \sqrt{\epsilon} t)-\delta u_{t}\right), \\
u(0, t)=u(\pi, t)=0=u_{x x}(0, t)=u_{x x}(\pi, t)
\end{gathered}
$$

where $u(x, t) \in \mathbb{R}$ is the transverse deflection of the axis of the beam; $\gamma \geq 0$ is an external load, $\kappa>0$ is a ratio indicating the external rigidity and $\delta>0$ is the damping, $\epsilon$ and $\nu$ are small parameters, the function $h(x, t)$ represents the

1991 Mathematics Subject Classification. 35, 37.

Key words and phrases. Compressed beam, chaotic solutions, bifurcation.

FB partially supported by G.N.A.M.P.A. - INdAM (Italy) and MURST (Italy).

MFe partially supported by MURST (Italy) and Grant GA-MS 1/6179/01.

MFr partially supported by G.N.A.M.P.A. - INdAM (Italy).

(C)2007 International Press 
periodic (in time) forcing that is distributed along the whole beam. We assume that $h \in L^{\infty}\left(\mathbb{R}, L^{2}([0, \pi])\right)$ is a 1 -periodic function of $t$ with $\left\|\int_{0}^{\pi} h(x, t)^{2} d x\right\|_{\infty}=1$. Therefore $\epsilon \nu$ represents the strength of the forcing.

The first work on oscillations of an elastic beam subject to an axial compression was done by Holmes and Marsden [5]. More recent works on the full equation are due to Rodrigues and Silveira [14] and Berti and Carminati [2]. An undamped buckled beam is investigated by Yagasaki $[\mathbf{1 9}]$ to show Arnold diffusion type motions.

The above papers discuss equation (1) when the external load $\gamma$ is not resonant and $\kappa \in \mathbb{R}$ is fixed. Here we discuss the complementary case. Precisely we assume that $\gamma$ is slightly larger than the $i$-th eigenvalue of the unperturbed problem: $\gamma=$ $i^{2}+\epsilon \sigma^{2}$, where $i \in \mathbb{N}$ is fixed, $\epsilon>0$ and $\sigma \in(0,1]$. Therefore we will also assume that $\kappa=\epsilon k$, so that the contribution given from the stress due to the external rigidity, does not drive the system too far away from the resonance.

Next we briefly summarize the status on chaos in partial differential equations. For the complex Ginzburg-Landau equation in the near nonlinear Schrödinger regime (i.e. perturbed nonlinear Schrödinger equation), existence of homoclinic orbits was proved by Li, McLaughlin, Shatah and Wiggins $[\mathbf{6}, \mathbf{1 2}, \mathbf{1 3}]$, and existence of chaos was proved by $\mathrm{Li}[\mathbf{7}, \mathbf{8}]$ under generic conditions. For perturbed sineGordon equation, existence of chaos and chaos cascade around a homoclinic tube was proved by $\mathrm{Li}[\mathbf{9}, \mathbf{1 0}, \mathbf{1 1}]$. For the reaction-diffusion equation, entropy study on the complexity of attractor was conducted by Zelik $[\mathbf{1 5}, \mathbf{1 6}, \mathbf{1 7}]$. Chaotic oscillations of a linear wave equation with nonlinear boundary conditions are shown by Chen, Hsu and Zhou [3]. The development on chaos and its controlling for PDEs is summarized by Zhao [18].

The plan of our paper is as follows. In Section 2, we formulate weak solutions to (1). Then in Section 3 we prove the main result Theorem 3 of this paper by using some ideas of $[\mathbf{1}]$.

\section{Formulation of weak solutions}

It is easily observed that the unperturbed problem

$$
\begin{aligned}
& u_{x x x x}+\gamma u_{x x}=0, \\
& u(0, t)=u(\pi, t)=0=u_{x x}(0, t)=u_{x x}(\pi, t),
\end{aligned}
$$

admits $\left\{j^{2} \mid j \in \mathbb{N}\right\}$ as set of eigenvalues and that the corresponding eigenfunctions $\sqrt{\frac{2}{\pi}} \sin (j x)$, where $j \in \mathbb{N}$, form an orthonormal system in $L^{2}([0, \pi])$ which generates the space $W=H_{0}^{2}([0, \pi])$.

First of all we make the linear scale $t \leftrightarrow \sqrt{\epsilon} t$. Then equations (1), (2) read:

$$
\begin{aligned}
& u_{t t}+\frac{1}{\epsilon}\left[u_{x x x x}+\left(i^{2}+\epsilon \sigma^{2}\right) u_{x x}\right]-k f\left(\int_{0}^{\pi} u_{x}^{2}(\xi, t) d \xi\right) u_{x x}=\nu h(x, t)-\sqrt{\epsilon} \delta u_{t} \\
& u(0, t)=u(\pi, t)=0=u_{x x}(0, t)=u_{x x}(\pi, t)
\end{aligned}
$$


We want to solve (3) in a weak form, that is we look for a function $u \in \tilde{U}:=$ $L^{\infty}(\mathbb{R}, W) \subset L^{\infty}([0, \pi] \times \mathbb{R})$ such that

(4)

$$
\begin{gathered}
\int_{-\infty}^{+\infty} \int_{0}^{\pi} u(x, t)\left\{\Psi_{t t}+\frac{1}{\epsilon}\left[\Psi_{x x x x}+\left(i^{2}+\epsilon \sigma^{2}\right) \Psi_{x x}\right]-k f\left(\int_{0}^{\pi} u_{x}^{2}(\xi, t) d \xi\right) \Psi_{x x}+\right. \\
\left.-\sqrt{\epsilon} \delta \Psi_{t}-\nu \Psi(x, t) h(x, t)\right\} d x d t=0
\end{gathered}
$$

for any $\Psi(x, t) \in \mathcal{C}^{\infty}([0, \pi] \times \mathbb{R})$ with compact support and such that

$$
\Psi(0, t)=\Psi(\pi, t)=\Psi_{x x}(0, t)=\Psi_{x x}(\pi, t)=0 .
$$

\section{Chaotic Solutions}

In this section we prove the existence of chaotic solutions for (1). The plan is as follows: first by using a Galerkin method, we rewrite (4) as an infinite system of differential equations (5)-(7). Then following [1], we apply a Ljapunov-Schmidt reduction method for this system to derive a Melnikov function. The existence of its simple roots predicts chaos for (1) (see Theorem 3).

To start with, note that we can expand the function $u(x, t) \in \tilde{U}$ as follows

$$
u(x, t)=\sqrt{\frac{2}{\pi}}\left[\sum_{0<l<i} \phi_{l}(t) \sin (l x)+y(t) \sin (i x)+\sum_{j>i} z_{j}(t) \sin (j x)\right],
$$

where $\phi_{l}(t), y(t), z_{j}(t) \in L^{\infty}(\mathbb{R})$, the expansion holding in $H_{0}^{2}([0, \pi])$. Similarly we write:

$$
\Psi(x, t)=\sqrt{\frac{2}{\pi}}\left[\sum_{l=1}^{i-1} \psi_{l}(t) \sin (l x)+\psi_{i}(t) \sin (i x)+\sum_{j=i+1}^{\infty} \psi_{j}(t) \sin (j x)\right],
$$

where, for any $k \geq 1, \psi_{k}(t) \in C_{0}^{\infty}(\mathbb{R})$, the space of $C^{\infty}$-functions on $\mathbb{R}$ having compact supports. Plugging the above expression for $u(x, t)$ and $\Psi(x, t)$ into (4) and using the orthonormality, we arrive at the system of equations for the components $\left(\phi_{l}(t), y(t), z_{j}(t)\right)$ of $u(x, t)$

$$
\begin{aligned}
& \ddot{\phi}_{l}(t)-\frac{i^{2}-l^{2}+\epsilon \sigma^{2}}{\epsilon} l^{2} \phi_{l}(t)+k l^{2} f\left(\sum_{0<l<i} l^{2} \phi_{l}(t)^{2}+i^{2} y^{2}(t)+\sum_{j>i} j^{2} z_{j}(t)^{2}\right) \phi_{l}(t) \\
& +\sqrt{\epsilon} \delta \dot{\phi}_{l}(t)-\nu \sqrt{\frac{2}{\pi}} \int_{0}^{\pi} h(x, t) \sin (l x) d x=0
\end{aligned}
$$

$$
\begin{aligned}
& \ddot{y}(t)-\sigma^{2} i^{2} y(t)+k i^{2} f\left(\sum_{0<l<i} l^{2} \phi_{l}^{2}(t)+i^{2} y^{2}(t)+\sum_{j>i} j^{2} z_{j}^{2}(t)\right) y(t)+\sqrt{\epsilon} \delta \dot{y}(t) \\
& -\nu \sqrt{\frac{2}{\pi}} \int_{0}^{\pi} h(x, t) \sin (i x) d x=0
\end{aligned}
$$

$$
\begin{aligned}
& \ddot{z}_{j}(t)+\frac{j^{2}-i^{2}-\epsilon \sigma^{2}}{\epsilon} j^{2} z_{j}(t)+k j^{2} f\left(\sum_{0<l<i} l^{2} \phi_{l}(t)^{2}+i^{2} y^{2}(t)+\sum_{j>i} j^{2} z_{j}(t)^{2}\right) z_{j}(t) \\
& +\sqrt{\epsilon} \delta \dot{z}_{j}(t)-\nu \sqrt{\frac{2}{\pi}} \int_{0}^{\pi} h(x, t) \sin (j x) d x=0
\end{aligned}
$$

where $0<l<i<j$. This way we have decomposed the problem along three submanifolds: a strongly hyperbolic second order problem in $\mathbb{R}^{i-1}$, a hyperbolic second order problem in $\mathbb{R}$, and a second order problem in an infinite dimensional center manifold. 
To simplify matter we replace $\left(\phi_{l}(t), y(t), z_{j}(t)\right)$ with $\left(i \phi_{l}(t / i), i y(t / i), i z_{j}(t / i)\right)$. Then, writing again $\left(\phi_{l}(t), y(t), z_{j}(t)\right)$ for $\left(i \phi_{l}(t / i), i y(t / i), i z_{j}(t / i)\right)$ equations (5)(7) read:

$$
\begin{aligned}
& \ddot{\phi}_{l}(t)-\frac{i^{2}-l^{2}+\epsilon \sigma^{2}}{\epsilon}\left(\frac{l}{i}\right)^{2} \phi_{l}(t) \\
& \quad+k\left(\frac{l}{i}\right)^{2} f\left(\sum_{0<l<i}\left(\frac{l}{i}\right)^{2} \phi_{l}(t)^{2}+y^{2}(t)+\sum_{j>i}\left(\frac{j}{i}\right)^{2} z_{j}(t)^{2}\right) \phi_{l}(t) \\
& \quad+\sqrt{\epsilon} \delta i^{-1} \dot{\phi}_{l}(t)-\nu i^{-1} \sqrt{\frac{2}{\pi}} \int_{0}^{\pi} h(x, t / i) \sin (l x) d x=0 \\
& \ddot{y}(t)-\sigma^{2} y(t)+k f\left(\sum_{0<l<i}\left(\frac{l}{i}\right)^{2} \phi_{l}^{2}(t)+y^{2}(t)+\sum_{j>i}\left(\frac{j}{i}\right)^{2} z_{j}^{2}(t)\right) y(t) \\
& +\sqrt{\epsilon} \delta i^{-1} \dot{y}(t)-\nu i^{-1} \sqrt{\frac{2}{\pi}} \int_{0}^{\pi} h(x, t / i) \sin (i x) d x=0 \\
& \quad \ddot{z}_{j}(t)-i^{2}-j^{2}+\epsilon \sigma^{2} \\
& \left.\quad+k\left(\frac{j}{i}\right)^{2}{ }^{2} z_{j} \sum_{0<l<i}\left(\frac{l}{i}\right)^{2} \phi_{l}(t)^{2}+y^{2}(t)+\sum_{j>i}\left(\frac{j}{i}\right)^{2} z_{j}(t)^{2}\right) z_{j}(t) \\
& \quad+\sqrt{\epsilon} \delta i^{-1} \dot{z}_{j}(t)-\nu i^{-1} \sqrt{\frac{2}{\pi}} \int_{0}^{\pi} h(x, t / i) \sin (j x) d x=0
\end{aligned}
$$

Let $C_{b}^{1}(I)$ be the Banach space of $C^{1}$-functions on the interval $I \subset \mathbb{R}$ that are bounded together with their first derivative with the norm

$$
\|f\|_{C_{b}^{1}(I)}:=\|f\|_{\infty}+\|\dot{f}\|_{\infty} .
$$

Let $k$ be a positive integer and set $m=k i$. In the following we will need to consider the Banach space of piecewise $C^{1}$ functions on $\mathbb{R}$ with possible jumps (of the function or its derivative) at the points $(2 j-1) m, j \in \mathbb{Z}$ and such that the $\sup _{j \in \mathbb{Z}}\left\{\|y\|_{C_{b}^{1}([(2 j-1) m,(2 j+1) m])}\right\}<\infty$. We use the shorthand

$$
Y:=\cap_{j \in \mathbb{Z}} C_{b}^{1}([(2 j-1) m,(2 j+1) m])
$$

to denote this Banach space with the norm

$$
\|y\|_{Y}:=\sup _{j \in \mathbb{Z}}\left\{\|y\|_{C_{b}^{1}([(2 j-1) m,(2 j+1) m])}\right\}
$$

We also introduce the following Banach spaces:

$$
\begin{aligned}
& Z:=\left\{z(t)=\left\{z_{j}(t)\right\}_{j>i} \mid z_{j} \in C_{b}^{0}(\mathbb{R}),\left\|\sum_{j>i} j^{4} z_{j}^{2}(t)\right\|_{\infty}<\infty\right\}, \\
& \Phi:=\left\{\phi(t)=\left\{\phi_{l}(t)\right\}_{0<l<i} \mid \phi_{l}(t) \in C_{b}^{1}(\mathbb{R})\right\}, \\
& U:=\{u(t)=(\phi(t), y(t), z(t)) \mid \phi(t) \in \Phi, y(t) \in Y \text { and } z(t) \in Z\}
\end{aligned}
$$

endowed respectively with the norm

$$
\begin{aligned}
\|z\|_{Z} & :=\left\|\sqrt{\sum_{j>i} j^{4} z_{j}^{2}(t)}\right\|_{\infty}, \quad\|\phi\|_{\Phi}:=\sqrt{\sum_{0<l<i}\left\|\phi_{l}\right\|_{C_{b}^{1}}^{2}}, \quad \text { and } \\
\|u\|_{U} & :=\|\phi\|_{\Phi}+\|y\|_{Y}+\|z\|_{Z}
\end{aligned}
$$

where obviously $u(t)=(\phi(t), y(t), z(t))$. Observe that $U \subset \tilde{U}$ therefore we will in fact work in $U$. 
We assume that $f(x)$ satisfies the following:

\section{Hypotheses}

F1: The function $f: \mathbb{R}_{0}^{+} \rightarrow \mathbb{R}_{0}^{+} \in C([0, \infty)) \cap C^{2}((0, \infty))$. Moreover we assume the following conditions hold: $f(0)=0, \limsup \left|x f^{\prime}\left(x^{2}\right)\right|<\infty$ and $\limsup _{x \rightarrow 0^{+}}\left|x^{3} f^{\prime \prime}\left(x^{2}\right)\right|<\infty$.

F2: The equation

$$
\ddot{y}-\sigma^{2} y+k f\left(y^{2}\right) y=0
$$

has a positive homoclinic solution that is a $C^{2}$-solution $\gamma(t)>0$ such that $\lim _{|t| \rightarrow \infty} \gamma(t)=\lim _{|t| \rightarrow \infty} \dot{\gamma}(t)=0$.

1. REmark. i) Observe that $\gamma_{i}(t)=\gamma(i t) / i$ solves the equation

$$
\ddot{y}-i^{2} \sigma^{2} y+k i^{2} f\left(i^{2} y^{2}\right) y=0
$$

for any $i \in \mathbb{N} \backslash\{0\}$. That is $\gamma_{i}(t)$ is a solution of the equation obtained from (6) taking $\phi_{l}(t)=0, z_{j}(t)=0$ and $\epsilon=\nu=0$. We will refer to equation (12) as the unperturbed problem.

ii) Equation (11) has the energy function

$$
E(y, \dot{y})=\dot{y}^{2}+\int_{0}^{y^{2}}\left(k f(s)-\sigma^{2}\right) \mathrm{d} s
$$

which is even in both $y$ and $\dot{y}$. Since $\lim _{t \rightarrow \infty} \gamma(t)=0$, we see that $\dot{\gamma}(t)=0$ has a solution $t_{0}$. It can be proved (see [4]) that this solution is unique. Hence we can assume $t_{0}=0$ and then $\gamma(t)=\gamma(-t)$ because of uniqueness. Thus either $\gamma(t)$ has a positive maximum or it has a negative minimum at the point $t=0$. Since $-\gamma(t)$ satisfies equation (11) when $\gamma(t)$ does, we see that the assumption $\gamma(t)>0$ is not restrictive. Then, $\gamma(t)$ is increasing on $(-\infty, 0]$ and decreasing on $[0, \infty)$. As a consequence $0 \leq \gamma(t) \leq M:=\gamma(0)$. Since the energy function $E(y, \dot{y})$ is constant along $(\gamma(t), \dot{\gamma}(t))$ and $\dot{\gamma}(0)=0$ we get

$$
\int_{0}^{M^{2}}\left(k f(s)-\sigma^{2}\right) \mathrm{d} s=0
$$

(note that $\lim _{t \rightarrow \infty} E(\gamma(t), \dot{\gamma}(t))=E(0,0)=0$ ) and

$$
\int_{0}^{x^{2}}\left(k f(s)-\sigma^{2}\right) \mathrm{d} s<0
$$

for $0<x<M$. Finally $k f\left(M^{2}\right) \neq \sigma^{2}$, since, otherwise $x=M$ would be a fixed point of equation (11). As a matter of fact we have $k f\left(M^{2}\right)>\sigma^{2}$, since the function

$$
\int_{0}^{x^{2}}\left(k f(s)-\sigma^{2}\right) \mathrm{d} s
$$

passes from negative values to 0 when $x \rightarrow M^{-}$and then its derivative at $x=M$ must be non negative. As a consequence assumption F2 implies that the following condition holds: 
F2': There exists $M>0$ such that $\int_{0}^{x^{2}}\left[k f(s)-\sigma^{2}\right] d s<0$ for any $0<x<M$ and $\int_{0}^{M^{2}}\left[k f(s)-\sigma^{2}\right] d s=0$. Moreover $k f\left(M^{2}\right)>\sigma^{2}$.

On the other hand if condition F2' holds then the solution $\gamma(t)$ of (11) such that $\gamma(0)=M$ and $\dot{\gamma}(0)=0$ satisfies $0<\gamma(t)<M$ for any $t \neq 0$, and is homoclinic to the (hyperbolic) fixed point $x=0, \dot{x}=0$ of (11). Thus the two conditions $\mathbf{F} 2$ and F2' are equivalent.

Finally we observe that the curve $(\gamma(t), \dot{\gamma}(t))$ is contained in the sector $\{(y, \dot{y}) \mid y \geq$ 0 and $|\dot{y}| \leq \sigma y\}$, that is:

$$
|\dot{\gamma}(t)| \leq \sigma \gamma(t)
$$

for any $t \in \mathbb{R}$.

iii) Since we look for solutions which are close to the homoclinic orbit, in fact it is enough that $f$ is defined just for $0 \leq x \leq M^{2}+1$.

iv) Assumption F1 is satisfied in particular if we take any function $f(x)$ of the form $f(x)=g\left(x^{\alpha}\right)$, where $\alpha \geq \frac{1}{2}$ and $g(x) \in C^{2}([0, \infty),[0, \infty))$ is a positive function such that $g(0)=0$.

v) From $\mathbf{F} 1$ it follows that:

$$
\lim _{x \rightarrow 0^{+}} x f^{\prime}(x)=\lim _{x \rightarrow 0} x^{2} f^{\prime}\left(x^{2}\right)=0
$$

and similarly

$$
\lim _{x \rightarrow 0^{+}} x^{2} f^{\prime \prime}(x)=\lim _{x \rightarrow 0} x^{4} f^{\prime \prime}\left(x^{2}\right)=0 .
$$

Hence the function $x f\left(x^{2}\right)$ is $C^{1}$ on $\mathbb{R}$ and its second derivative is bounded on $K \backslash\{0\}, K$ being any fixed compact subset of $\mathbb{R}$. In fact, for $x \neq 0$, we have

$$
\frac{d}{d x}\left[x f\left(x^{2}\right)\right]=2 x^{2} f^{\prime}\left(x^{2}\right)+f\left(x^{2}\right) \rightarrow 0=\frac{d}{d x}\left[x f\left(x^{2}\right)\right]_{\mid x=0}
$$

as $x \rightarrow 0$ (see point $\mathrm{v})$ ). Thus $\frac{d}{d x}\left[x f\left(x^{2}\right)\right]$ is continuous in $\mathbb{R}$. Next

$$
\frac{d^{2}}{d x^{2}}\left[x f\left(x^{2}\right)\right]=6 x f^{\prime}\left(x^{2}\right)+4 x^{3} f^{\prime \prime}\left(x^{2}\right)
$$

is bounded on $K \backslash\{0\}$ for any, given, compact subset $K$ of $\mathbb{R}$ because of assumption F1.

It follows from Remark 1-v) that the functions $f(x), x f^{\prime}(x), x^{2} f^{\prime \prime}(x)$ are uniformly continuous in any compact interval $\left[0, M_{0}\right]$. We set

$$
\begin{aligned}
& N:=\max \left\{f\left(x^{2}\right) \mid x \in\left[0, \sqrt{M^{2}+1}\right]\right\}, \\
& N^{\prime}:=\sup \left\{2\left|x f^{\prime}\left(x^{2}\right)\right| \mid x \in\left(0, \sqrt{M^{2}+1}\right]\right\}, \\
& N^{\prime \prime}:=\sup \left\{\left|x^{3} f^{\prime \prime}\left(x^{2}\right)\right| \mid x \in\left(0, \sqrt{M^{2}+1}\right]\right\} .
\end{aligned}
$$

where, we recall, $M=\gamma(0)$, and:

$$
F(\eta, \zeta)=f\left(\eta^{2}+\zeta^{2}\right) \eta
$$


Then the following result hold:

1. Lemma. We have $F(\eta, \zeta) \in C^{1}\left(\mathbb{R}^{2}\right) \cap C^{2}\left(\mathbb{R}^{2} \backslash\{(0,0)\}\right)$ and, for any compact subset $K \subset \mathbb{R}^{2}$, the second derivatives of $F(\eta, \zeta)$ are bounded on $K \backslash\{(0,0)\}$. Moreover $F_{\zeta}(\eta, 0)=F_{\zeta}(0, \zeta)=F_{\eta}(0,0)=0$. More precisely

$$
\left|F_{\eta}(\eta, \zeta)\right| \leq N^{\prime}\left(|\eta|+\sqrt{\eta^{2}+\zeta^{2}}\right)
$$

, and $\left|F_{\zeta}(\eta, \zeta)\right| \leq N^{\prime} \min \{|\eta|,|\zeta|\}$ for any $(\eta, \zeta)$ such that $\eta^{2}+\zeta^{2} \leq M^{2}+1$.

Proof. Only the estimate $\left|F_{\eta}(\eta, \zeta)\right| \leq N^{\prime}\left(|\eta|+\sqrt{\eta^{2}+\zeta^{2}}\right)$ needs to be proved. For any $0<x \leq \sqrt{M^{2}+1}$ we have:

$$
f\left(x^{2}\right)=f\left(x^{2}\right)-f(0)=\int_{0}^{x} 2 t f^{\prime}\left(t^{2}\right) d t \leq N^{\prime} x .
$$

Then

$$
\left|F_{\eta}(\eta, \zeta)\right| \leq\left|2 \eta f^{\prime}\left(\eta^{2}+\zeta^{2}\right)\right||\eta|+\left|f\left(\eta^{2}+\zeta^{2}\right)\right| \leq N^{\prime}\left(|\eta|+\sqrt{\eta^{2}+\zeta^{2}}\right) .
$$

In the following we will make use of the following constants:

$$
\begin{aligned}
& k_{1}=\sup \left\{\left|F_{\zeta \zeta}(\eta, \zeta)\right|: 0<\eta^{2}+\zeta^{2} \leq M^{2}+1\right\}, \\
& k_{2}=\sup \left\{\left|F_{\eta \eta}(\eta, \zeta)\right|: 0<\eta^{2}+\zeta^{2} \leq M^{2}+1\right\}, \\
& \left.k_{3}=\sup \left\{\left|F_{\eta \zeta}(\eta, \zeta)\right|: 0<\eta^{2}+\zeta^{2} \leq M^{2}+1\right\}\right\} .
\end{aligned}
$$

It is easy to check that the constants $k_{1}, k_{2}$ and $k_{3}$ can be estimated in term of $N$, $N^{\prime}$ and $N^{\prime \prime}$. We leave these computations to the reader.

We begin by solving (6) for any fixed $\phi, z$ such that $\|\phi\|_{\Phi}<\rho,\|z\|_{Z}<\rho$, through the Banach Fixed Point Theorem. We will adapt to this setting the argument given in $[\mathbf{1}]$. Let $m \geq\left[\epsilon^{-3 / 4}\right]+1,\left[\epsilon^{-3 / 4}\right]$ being the integer part of $\epsilon^{-3 / 4}$. From now on we assume that $0<\epsilon \leq(1 / 2)^{4 / 3}$ so that $m \geq 3$. We look for chaotic patterns close to the homoclinic solution $\gamma_{i}(t) \sin (i x)$ of the unperturbed problem. Thus writing

$$
\sqrt{\frac{\pi}{2}} i u(x, t / i)=\sum_{l=1}^{i-1} \phi_{l}(t) \sin (l x)+y(t) \sin (i x)+\sum_{j=i+1}^{\infty} z_{j}(t) \sin (j x)
$$

we see that the components $\left(\phi_{l}(t), y(t), z_{j}(t)\right)$ solve (8)-(10) and $i u(x, t / i)$ is close to $\gamma(t) \sin (i x)$. Hence we introduce the following metric spaces. Let $\mathcal{E}=\{0,1\}^{\mathbb{Z}}$ be the space of doubly infinite sequences of 0's and 1's. Thus $E \in \mathcal{E}$ if and only if $E=\left\{e_{j}\right\}_{j \in \mathbb{Z}}$, with either $e_{j}=0$ or $e_{j}=1$. In $\mathcal{E}$ we take the norm

$$
\left\|\left\{e_{j}\right\}_{j \in \mathbb{Z}}\right\|=\sum_{j \in \mathbb{Z}} \frac{e_{j}}{2^{|j|}}
$$

moreover for any $E=\left\{e_{j}\right\}_{j \in \mathbb{Z}} \in \mathcal{E}$, we put

$$
\ell_{E}^{\infty}=\left\{\alpha:=\left\{\alpha_{j}\right\}_{j \in \mathbb{Z}} \in \ell^{\infty}(\mathbb{R}) \mid \alpha_{j} \in \mathbb{R} \text { and } \alpha_{j}=0 \text { if } e_{j}=0\right\},
$$


$\ell^{\infty}(\mathbb{R})$ being the Banach space of bounded, doubly infinity sequences of real numbers, endowed with the sup-norm. We will also consider the closed and bounded subset of $\mathcal{E} \times \ell^{\infty}(\mathbb{R})$ :

$$
X=\left\{(E, \alpha) \in \mathcal{E} \times \ell^{\infty}(\mathbb{R}) \mid \alpha \in \ell_{E}^{\infty} \quad \text { and } \quad\|\alpha\| \leq 2\right\}
$$

In $[\mathbf{1}$, p. 178] it has been proved that $X$ is closed.

For any $\xi=(E, \alpha) \in X$ we take the function $\gamma_{\xi}=\gamma_{(E, \alpha)} \in L^{\infty}(\mathbb{R})$ defined as

$$
\gamma_{\xi}(t)= \begin{cases}\gamma\left(t-2 j m-\alpha_{j}\right) & \text { if }(2 j-1) m<t \leq(2 j+1) m \text { and } e_{j}=1 \\ 0 & \text { if }(2 j-1) m<t \leq(2 j+1) m \text { and } e_{j}=0 .\end{cases}
$$

For sake of simplicity we will silently include, in the above definitions, also the end points of the intervals $[(2 j-1) m,(2 j+1) m], j \in \mathbb{Z}$. From [1, p. 178] it follows that $\gamma_{\xi}(t)$ has the following properties

(i): $\gamma_{\xi}(t)$ is a bounded, piecewise $C^{2}$-function, with bounded derivatives and possible jumps at the points $(2 j-1) m, j \in \mathbb{Z}$, and satisfies, in any of the intervals $((2 j-1) m,(2 j+1) m)$ the equation

$$
\ddot{y}+\left[k f\left(y^{2}\right)-\sigma^{2}\right] y=0 .
$$

Moreover $\gamma_{\xi}(t), \dot{\gamma}_{\xi}(t), \ddot{\gamma}_{\xi}(t)$ and are bounded uniformly with respect to $(\xi, m)$ and the following estimates hold:

$$
\left\|\gamma_{\xi}\right\|_{\infty} \leq M,\left\|\dot{\gamma}_{\xi}\right\|_{\infty} \leq \sigma M,\left\|\ddot{\gamma}_{\xi}\right\|_{\infty} \leq M\left[\sigma^{2}+k N\right] .
$$

(ii): $\gamma_{\xi}(t), \dot{\gamma}_{\xi}(t), \ddot{\gamma}_{\xi}(t)$ are Lipschitz continuous in $\alpha \in \ell_{E}^{\infty}$ uniformly with respect to $(E, m)$. Actually we have:

$$
\begin{aligned}
& \left\|\gamma_{\xi^{\prime}}-\gamma_{\xi^{\prime \prime}}\right\|_{\infty} \leq \sigma M\left\|\alpha^{\prime}-\alpha^{\prime \prime}\right\| \\
& \left\|\dot{\gamma}_{\xi^{\prime}}-\dot{\gamma}_{\xi^{\prime \prime}}\right\|_{\infty} \leq M\left[\sigma^{2}+k N\right]\left\|\alpha^{\prime}-\alpha^{\prime \prime}\right\| \\
& \left\|\ddot{\gamma}_{\xi^{\prime}}-\ddot{\gamma}_{\xi^{\prime \prime}}\right\|_{\infty} \leq\left(\sigma^{2}+k N^{\prime}\right) \sigma M\left\|\alpha^{\prime}-\alpha^{\prime \prime}\right\| .
\end{aligned}
$$

Since $\gamma(t)$ is a solution of (11) that tends to $y=0$ as $|t| \rightarrow \infty$, it follows from standard theory that there is a constant $A_{1}>0$ such that

$$
\left|\gamma_{\xi}(t)\right| \leq A_{1} e^{-\sigma\left|t-2 j m-\alpha_{j}\right|}, \quad t \in((2 j-1) m,(2 j+1) m] .
$$

From $\mathbf{F} 1$ it follows that $\dot{\gamma}(t)$ is a bounded solution of the variational problem associated to $\ddot{y}+\left[k f\left(y^{2}\right)-\sigma^{2}\right] y=0$. More precisely all bounded solutions of

$$
\ddot{y}+\left[2 k f^{\prime}\left(\gamma^{2}(t)\right) \gamma^{2}(t)+k f\left(\gamma^{2}(t)\right)-\sigma^{2}\right] y=0
$$

take the form $c \dot{\gamma}(t)$ where $c \in \mathbb{R}$. We now study the problem of existence of solutions $y(t) \in W^{2, \infty}(\mathbb{R})$ of the following linear non-homogeneous equation

$$
\begin{aligned}
& \ddot{y}+\left[2 k f^{\prime}\left(\gamma_{\xi}^{2}(t)\right) \gamma_{\xi}^{2}(t)+k f\left(\gamma_{\xi}^{2}(t)\right)-\sigma^{2}\right] y=h(t) \\
& y\left((2 j+1) m_{+}\right)-y\left((2 j+1) m_{-}\right)=\Gamma_{j} \\
& \dot{y}\left((2 j+1) m_{+}\right)-\dot{y}\left((2 j+1) m_{-}\right)=\Gamma_{j}^{\prime} \\
& \dot{y}\left(2 j m+\alpha_{j}\right)=0, \quad \text { for any } j \in \mathbb{Z} \text { such that } e_{j}=1
\end{aligned}
$$

where $h \in L^{\infty}(\mathbb{R})$, and the ordinary differential equation in (20) holds almost everywhere.

We note that

$$
\left[k f\left(y^{2}\right)-\sigma^{2}\right] y \in C^{1}(\mathbb{R})
$$


and $\left.\frac{\mathrm{d}}{\mathrm{d} y}\left[k f\left(y^{2}\right)-\sigma^{2}\right] y\right|_{y=0}=-\sigma^{2}<0$ (see Remark 1-v)). Thus, adapting to this setting the argument given in $[\mathbf{1}$, Lemma 1] with few small changes, we obtain the following result:

2. Lemma. There exist positive constants $A, B, C \in \mathbb{R}$ and $m_{0} \in \mathbb{N}$ such that for any $\xi=(E, \alpha) \in X, m \geq m_{0}$, and $j \in \mathbb{Z}$, there exist linear functionals $\mathcal{L}_{m, \xi, j}$ : $L^{\infty}(\mathbb{R}) \times \ell^{\infty}(\mathbb{R}) \times \ell^{\infty}(\mathbb{R}) \rightarrow \mathbb{R}$, such that $\left\|\mathcal{L}_{m, \xi, j}\right\| \leq A e_{j} e^{-\sigma m}$, and with the property that if $h \in L^{\infty}(\mathbb{R}), \Gamma=\left\{\Gamma_{j}\right\}_{j \in \mathbb{Z}}, \Gamma^{\prime}=\left\{\Gamma_{j}^{\prime}\right\}_{j \in \mathbb{Z}} \in \ell^{\infty}(\mathbb{R})$ then (20) has a unique solution $y(t, \xi)$ which is bounded on $\mathbb{R}$ and $C^{1}$ in any interval $((2 j-1) m,(2 j+1) m)$ if and only if

$$
\mathcal{L}_{m, \xi, j} h+\int_{(2 j-1) m}^{(2 j+1) m} \dot{\gamma}_{\xi}(t) h(t) d t=0
$$

for any $j \in \mathbb{Z}$. Moreover, the following properties hold:

: i)

$$
\left\|y_{1}(\cdot, \xi)\right\|_{\infty}+\left\|\dot{y}_{1}(\cdot, \xi)\right\|_{\infty} \leq B\left[\|h\|_{\infty}+\|\Gamma\|+\sigma^{-1}\left\|\Gamma^{\prime}\right\|\right]
$$

: ii) let $y_{p}(t)$ be the unique bounded solution, $C^{1}$ on $((2 j-1) m,(2 j+1) m)$, of the equation

$$
\begin{aligned}
& \ddot{y}-\sigma^{2} y=h(t), \\
& y\left((2 j+1) m_{+}\right)-y\left((2 j+1) m_{-}\right)=\Gamma_{j} \\
& \dot{y}\left((2 j+1) m_{+}\right)-\dot{y}\left((2 j+1) m_{-}\right)=\Gamma_{j}^{\prime}
\end{aligned}
$$

then there exists a constant $C$ such that

$$
\left|y(t, \xi)-y_{p}(t)\right| \leq C\left(e^{-\sigma m / 2}+e^{-\sigma\left(t-2 j m-\alpha_{j}\right) / 2}\right)\|h\|_{\infty}
$$

for any $t \in((2 j-1) m,(2 j+1) m)$ and $j \in \mathbb{Z}$;

: iii) let $\xi^{\prime}=\left(E, \alpha^{\prime}\right), \xi^{\prime \prime}=\left(E, \alpha^{\prime \prime}\right)$ with $\alpha^{\prime}, \alpha^{\prime \prime} \in \ell_{E}^{\infty}(\mathbb{R})$ and $\xi$ be either $\xi^{\prime}$ or $\xi^{\prime \prime}$. Assume that $h(t, \xi) \in L^{\infty}(\mathbb{R})$ satisfies (21). Then there exists a constant $c_{1}$ independent of $\xi$ such that the following holds:

$$
\begin{aligned}
& \max \left\{\left\|y\left(\cdot, \xi^{\prime}\right)-y\left(\cdot, \xi^{\prime \prime}\right)\right\|_{\infty},\left\|\dot{y}\left(\cdot, \xi^{\prime}\right)-\dot{y}\left(\cdot, \xi^{\prime \prime}\right)\right\|_{\infty}\right\} \\
& \leq B\left\|h\left(t, \xi^{\prime}\right)-h\left(t, \xi^{\prime \prime}\right)\right\|_{\infty}+c_{1}\left\|h\left(t, \xi^{\prime \prime}\right)\right\|_{\infty}\left\|\alpha^{\prime}-\alpha^{\prime \prime}\right\| .
\end{aligned}
$$

Finally, for any $m \geq m_{0}$, the map $\mathcal{L}_{m}: X \times L^{\infty}(\mathbb{R}) \rightarrow \ell^{\infty}(\mathbb{R})$ defined as $\mathcal{L}_{m}(\xi, h)=$ $\left\{\mathcal{L}_{m, \xi, j} h\right\}_{j \in \mathbb{Z}}$ is Lipschitz in $\alpha \in \ell_{E}^{\infty}$ uniformly with respect to $(E, m)$.

In order to apply Lemma 2, we consider the set

$$
\mathcal{S}_{m, \xi}:=\left\{h \in L^{\infty}(\mathbb{R}) \mid \mathcal{L}_{m, \xi, j} h+\int_{(2 j-1) m}^{(2 j+1) m} \dot{\gamma}_{\xi}(t) h(t) d t=0 \text { for any } j \in \mathbb{Z}\right\} .
$$

Note that when $\xi=0$ (i.e. $(E, \alpha)=(0,0))$ we have $\mathcal{S}_{m, \xi}=L^{\infty}(\mathbb{R})$.

Following [1] we obtain this result

1. Proposition. There exists a projection $Q_{m, \xi}: L^{\infty}(\mathbb{R}) \rightarrow L^{\infty}(\mathbb{R})$ onto $\mathcal{S}_{m, \xi}$ which is uniformly bounded with respect to $(m, \xi)$ and Lipschitz in $\alpha \in \ell_{E}^{\infty}$ uniformly with respect to $(m, E)$, that is constants $A_{2}, L$, independent of $(m, E)$, exist such that

$$
\left\|Q_{m, \xi}\right\| \leq A_{2}
$$


and

$$
\left\|Q_{m,(E, \alpha)}-Q_{m,\left(E, \alpha^{\prime}\right)}\right\| \leq L\left\|\alpha-\alpha^{\prime}\right\|
$$

for any $m \geq \bar{m}$ and $(E, \alpha),\left(E, \alpha^{\prime}\right) \in X$.

According to Lemma 2 we define a linear bounded operator $V_{m, \xi}: \mathcal{S}_{m, \xi} \times$ $\ell^{\infty}(\mathbb{R}) \times \ell^{\infty}(\mathbb{R}) \rightarrow Y$, with $\left\|V_{m, \xi}\right\| \leq B$, where the norm on $\ell^{\infty}(\mathbb{R}) \times \ell^{\infty}(\mathbb{R})$ is defined as $\left\|\Gamma_{1}\right\|+\sigma^{-1}\left\|\Gamma_{2}\right\|$, such that $V_{m, \xi}\left(h, \Gamma, \Gamma^{\prime}\right)(t)$ is the unique weak solution of (20). Note that, from (22) and (24) we obtain the following. Given $h(t) \in \mathcal{S}_{m, \xi}$, $h_{1}(t) \in \mathcal{S}_{m, \xi_{1}}, h_{2}(t) \in \mathcal{S}_{m, \xi_{2}}, \Gamma, \Gamma_{1}, \Gamma_{2} \in \ell^{\infty}(\mathbb{R})$, then:

$$
\begin{aligned}
& \left\|V_{m, \xi_{2}}\left(h_{2}, \Gamma, \Gamma^{\prime}\right)-V_{m, \xi_{1}}\left(h_{1}, \Gamma, \Gamma^{\prime}\right)\right\|_{Y} \leq B\left\|h_{2}-h_{1}\right\|_{\infty}+c_{1}\left\|h_{2}\right\|_{\infty}\left\|\alpha_{2}-\alpha_{1}\right\| \\
& \left\|V_{m, \xi}\left(h, \Gamma_{2}, \Gamma_{2}^{\prime}\right)-V_{m, \xi}\left(h, \Gamma_{1}, \Gamma_{1}^{\prime}\right)\right\|_{Y} \leq B\left[\left\|\Gamma_{2}-\Gamma_{1}\right\|+\sigma^{-1}\left\|\Gamma_{2}^{\prime}-\Gamma_{1}^{\prime}\right\|\right]
\end{aligned}
$$

since $V_{m, \xi}\left(h, \Gamma_{2}, \Gamma_{2}^{\prime}\right)-V_{m, \xi}\left(h, \Gamma_{1}, \Gamma_{1}^{\prime}\right)$ is a bounded solution of

$$
\begin{aligned}
& \ddot{y}+\left[2 k f^{\prime}\left(\gamma_{\xi}^{2}(t)\right) \gamma_{\xi}^{2}(t)+k f\left(\gamma_{\xi}^{2}(t)\right)-\sigma^{2}\right] y=0, \\
& y\left((2 j+1) m_{+}\right)-y\left((2 j+1) m_{-}\right)=\Gamma_{2, j}-\Gamma_{1, j} \\
& \dot{y}\left((2 j+1) m_{+}\right)-\dot{y}\left((2 j+1) m_{-}\right)=\Gamma_{2, j}^{\prime}-\Gamma_{1, j}^{\prime}
\end{aligned}
$$

Note that, for the same reason,

$$
V_{m, \xi}\left(h, \Gamma_{2}, \Gamma_{2}^{\prime}\right)-V_{m, \xi}\left(h, \Gamma_{1}, \Gamma_{1}^{\prime}\right)=V_{m, \xi}\left(0, \Gamma_{2}-\Gamma_{1}, \Gamma_{2}^{\prime}-\Gamma_{1}^{\prime}\right) .
$$

We are looking for solutions of equations (8)-(10) such that the sup-norm of $y_{1}(t)-$ $\gamma_{\xi}(t), \phi_{l}(t)$ and $z_{j}(t)$ are small. Hence we replace $y(t)$ by $y(t)+\gamma_{\xi}(t)$ and project the right hand side of (9) obtained for the new $y(t)$ onto $\mathcal{S}_{m, \xi}$.

We set $\delta_{i}=\delta i^{-1}, \nu_{i}=\nu i^{-1}$ and

$$
\begin{aligned}
\mathfrak{F}(\xi, u, t) & =\mathfrak{F}(\xi, \phi, y, z, t) \\
& :=f\left[i^{-2} \sum_{l=1}^{i-1} l^{2} \phi_{l}^{2}(t)+\left(\gamma_{\xi}(t)+y(t)\right)^{2}+i^{-2} \sum_{j=i+1}^{\infty} j^{2} z_{j}^{2}(t)\right] .
\end{aligned}
$$

Then, recalling that $\dot{\gamma}(t)$ satisfies (19) and that $Q_{m, \xi} \dot{\gamma}_{\xi}=0$, we obtain the following system

$$
\begin{aligned}
\ddot{y}(t) & -\sigma^{2} y(t)+k\left[2 f^{\prime}\left(\gamma_{\xi}(t)^{2}\right) \gamma_{\xi}(t)^{2}+f\left(\gamma_{\xi}(t)^{2}\right)\right] y \\
& =-Q_{m, \xi}\left\{k \mathfrak{F}(\xi, u, t)\left[y(t)+\gamma_{\xi}(t)\right]-k f\left(\gamma_{\xi}(t)^{2}\right) \gamma_{\xi}(t)\right. \\
& -k\left[2 f^{\prime}\left(\gamma_{\xi}(t)^{2}\right) \gamma_{\xi}(t)^{2}+f\left(\gamma_{\xi}(t)^{2}\right)\right] y(t)+\sqrt{\epsilon} \delta_{i} \dot{y}(t) \\
& \left.-\nu_{i} \sqrt{\frac{2}{\pi}} \int_{0}^{\pi} h(x, t / i) \sin (i x) d x\right\} \\
\ddot{\phi}_{l}(t)- & \frac{i^{2}-l^{2}+\epsilon \sigma^{2}}{\epsilon}\left(\frac{l}{i}\right)^{2} \phi_{l}(t)+k\left(\frac{l}{i}\right)^{2} \mathfrak{F}(\xi, u, t) \phi_{l}(t) \\
+ & \sqrt{\epsilon} \delta_{i} \dot{\phi}_{l}(t)=\nu_{i} \sqrt{\frac{2}{\pi}} \int_{0}^{\pi} h(x, t / i) \sin (l x) \mathrm{d} x, \quad 0<l<i \\
\ddot{z}_{j}(t)+ & \frac{j^{2}-i^{2}-\epsilon \sigma^{2}}{\epsilon}\left(\frac{j}{i}\right)^{2} z_{j}(t)+k\left(\frac{j}{i}\right)^{2} \mathfrak{F}(\xi, u, t) z_{j}(t) \\
+ & \sqrt{\epsilon} \delta_{i} \dot{z}_{j}(t)=\nu_{i} \sqrt{\frac{2}{\pi}} \int_{0}^{\pi} h(x, t / i) \sin (j x) \mathrm{d} x, \quad j>i
\end{aligned}
$$


together with the jumping conditions

(33)

$$
\begin{aligned}
& y\left((2 k+1) m_{+}\right)-y\left((2 k+1) m_{-}\right)=\Gamma_{\xi, k}:=\gamma_{\xi}\left((2 k+1) m_{-}\right)-\gamma_{\xi}\left((2 k+1) m_{+}\right) \\
& \dot{y}\left((2 k+1) m_{+}\right)-\dot{y}\left((2 k+1) m_{-}\right)=\Gamma_{\xi, k}^{\prime}:=\dot{\gamma}_{\xi}\left((2 k+1) m_{-}\right)-\dot{\gamma}_{\xi}\left((2 k+1) m_{+}\right)
\end{aligned}
$$

for any $k \in \mathbb{Z}$. Of course, if $i=1$ the set of equations (31) is empty. To solve equation (30), (32) we want to use the Banach Fixed Point Theorem.

Let $\mathbb{N}_{m_{0}}:=\left\{m \in \mathbb{N}: m>m_{0}\right\}$. We define the operator $T^{2}(u, \xi, m, \epsilon)$ : $U \times X \times \mathbb{N}_{m_{0}} \times \mathbb{R}_{+} \rightarrow Y$ as follows

$$
T^{2}(u, \xi, m, \epsilon)=V_{m, \xi}\left(h(u, \xi, m, \epsilon ; t),\left\{\Gamma_{\xi, k}\right\}_{k \in \mathbb{Z}},\left\{\Gamma_{\xi, k}^{\prime}\right\}_{k \in \mathbb{Z}}\right)
$$

where $h$ is the right hand side of $(30), \Gamma_{\xi, k}, \Gamma_{\xi, k}^{\prime}$ are defined in (33) and $V_{m, \xi}$ is the linear map which associates to an element of $\mathcal{S}_{m, \xi} \times \ell^{\infty}(\mathbb{R}) \times \ell^{\infty}(\mathbb{R})$ the corresponding solution of (20). In the whole paper we will denote by $\Phi_{\rho}, Y_{\rho}$ and $Z_{\rho}$ the balls of radius $\rho>0$ centered at the origin, in the space $\Phi, Y$ and $Z$ respectively. We also set

$$
U_{\rho}=\Phi_{\rho} \times Y_{\rho} \times Z_{\rho} .
$$

Then we will define the operators $T^{1}(u, \xi, m, \epsilon)$ and $T^{3}(u, \xi, m, \epsilon)$ mapping $U_{\rho} \times$ $X \times \mathbb{N}_{m_{0}} \times \mathbb{R}_{+}$into $\Phi_{\rho}$ and $Z_{\rho}$ respectively, in such a way that the fixed points of the map $T(u, \xi, m, \epsilon):=\left(T^{1}(u, \xi, m, \epsilon), T^{2}(u, \xi, m, \epsilon), T^{3}(u, \xi, m, \epsilon)\right): U_{\rho} \times X \times$ $\mathbb{N}_{m_{0}} \times\left(0, \epsilon_{0}\right) \rightarrow U_{\rho}$ are solutions of (30)-(32) and we show that $T$ is a contraction in $U_{\rho}$ with contraction factor that tends to 0 when $\epsilon+\rho+1 / m$ tends to 0 .

We begin by proving that $T^{2}$ is well defined, that maps $U_{\rho}$ into $Y_{\rho}$, and that it is a contraction in $u \in U_{\rho}$ uniformly with respect to $(\xi, m, \epsilon)$. To reach this goal we write $T^{2}$ as the sum of two operators. Let:

$$
\begin{aligned}
h_{1}(y ; t) & =\sqrt{\epsilon} \delta_{i} \dot{y}(t)-\nu_{i} \sqrt{\frac{2}{\pi}} \int_{0}^{\pi} h(x, t / i) \sin (i x) \mathrm{d} x \\
h_{2}(\xi, u ; t) & =\mathfrak{F}(\xi, u, t)\left[\gamma_{\xi}(t)+y(t)\right]-f\left(\gamma_{\xi}(t)^{2}\right)\left[\gamma_{\xi}(t)+y(t)\right] \\
& -2 f^{\prime}\left(\gamma_{\xi}(t)^{2}\right) \gamma_{\xi}(t)^{2} y(t) \\
& =F\left(\gamma_{\xi}(t)+y(t), c(t)\right)-F\left(\gamma_{\xi}(t), 0\right)-F_{\eta}\left(\gamma_{\xi}(t), 0\right) y(t)
\end{aligned}
$$

where

$$
c(t)=i^{-1}\left[\sum_{l=1}^{i-1} l^{2} \phi_{l}^{2}(t)+\sum_{j=i+1}^{\infty} j^{2} z_{j}^{2}(t)\right]^{1 / 2}
$$

(see also (14), (29)). Note that

$$
\|c\|_{\infty}^{2} \leq\|\phi\|_{\Phi}^{2}+i^{-4}\|z\|_{Z}^{2}
$$

Now we define the operators

$$
\begin{aligned}
& T_{a}^{2}(\xi ; y):=-V_{m, \xi}\left(Q_{m, \xi} h_{1}(y ; \cdot),\left\{\Gamma_{\xi, k}\right\}_{k \in \mathbb{Z}},\left\{\Gamma_{\xi, k}^{\prime}\right\}_{k \in \mathbb{Z}}\right), \\
& T_{b}^{2}(\xi ; u):=-V_{m, \xi}\left(Q_{m, \xi} h_{2}(\xi, u ; \cdot), 0,0\right)
\end{aligned}
$$

and note that $T^{2}(u, \xi)=T_{a}^{2}(\xi ; y)+k T_{b}^{2}(\xi ; u)$. 
3. LEMMA. There are constants $\hat{k}_{1}>0, \hat{k}_{2}>0$ such that for any $\xi \in X$ and $y, \hat{y}, \bar{y} \in Y_{\rho}$ it results:

$$
\begin{aligned}
\left\|T_{a}^{2}(\xi ; y)\right\|_{Y} & \leq B\left\{A_{2}\left[\left|\nu_{i}\right|+\sqrt{\epsilon} \delta_{i}\|y\|_{Y}\right]+4 A_{1} e^{2} e^{-m \sigma}\right\} \\
\| T_{a}^{2}(\hat{\xi} ; \hat{y}) & -T_{a}^{2}(\bar{\xi} ; \bar{y})\left\|_{Y} \leq\left[\hat{k}_{1}\left(\left|\nu_{i}\right|+\delta_{i} \sqrt{\epsilon}\|\bar{y}\|_{Y}\right)+\hat{k}_{2} e^{-m \sigma}\right]\right\| \hat{\alpha}-\bar{\alpha} \| \\
& +A_{2} B \sqrt{\epsilon} \delta_{i}\|\hat{y}-\bar{y}\|_{Y}
\end{aligned}
$$

Proof. We begin by giving an estimate of $h_{1}(y ; t)$ and of $\Gamma_{k}, \Gamma_{k}^{\prime}$. ¿From Hölder inequality we get that

$$
\left|\sqrt{\frac{2}{\pi}} \int_{0}^{\pi} h(x, t / i) \sin (i x) d x\right| \leq \sup _{t \in \mathbb{R}}\|h(\cdot, t / i)\|_{2} \leq 1
$$

therefore

$$
\left\|h_{1}(y ; \cdot)\right\|_{\infty} \leq\left|\nu_{i}\right|+\delta_{i} \sqrt{\epsilon}\|y\|_{Y}
$$

Next, since (13) and (18) hold we get

$$
\left|\Gamma_{k}\right| \leq 2 A_{1} e^{2} e^{-m \sigma}, \quad\left|\Gamma_{k}^{\prime}\right| \leq 2 \sigma A_{1} e^{2} e^{-m \sigma} .
$$

Then the first inequality in (37) follows from Lemma 2 and (25). Next

$$
\begin{aligned}
\left\|T_{a}^{2}(\xi ; \hat{y})-T_{a}^{2}(\xi ; \bar{y})\right\|_{Y} & =\left\|\sqrt{\epsilon} \delta_{i} V_{m, \xi}\left(Q_{m, \hat{\xi}}[\dot{\hat{y}}-\dot{\bar{y}}], 0,0\right)\right\|_{Y} \\
& \leq A_{2} B \sqrt{\epsilon} \delta_{i}\|\hat{y}-\bar{y}\|_{Y} .
\end{aligned}
$$

Finally, recalling (25)-(28), we get

$$
\begin{aligned}
& \left\|T_{a}^{2}(\hat{\xi} ; y)-T_{a}^{2}(\bar{\xi} ; y)\right\|_{Y} \leq \\
& \leq\left\|V_{m, \hat{\xi}}\left(Q_{m, \hat{\xi}} h_{1}(y ; \cdot), \Gamma_{\hat{\xi}}, \Gamma_{\hat{\xi}}^{\prime}\right)-V_{m, \bar{\xi}}\left(Q_{m, \bar{\xi}} h_{1}(y ; \cdot), \Gamma_{\hat{\xi}}, \Gamma_{\hat{\xi}}^{\prime}\right)\right\|_{Y}+ \\
& +\left\|V_{m, \hat{\xi}}\left(0, \Gamma_{\hat{\xi}}-\Gamma_{\bar{\xi}}, \Gamma_{\hat{\xi}}^{\prime}-\Gamma_{\bar{\xi}}^{\prime}\right)\right\|_{Y} \leq \\
& \leq B\left\|Q_{m, \hat{\xi}}-Q_{m, \bar{\xi}}\right\|\left\|h_{1}(y ; \cdot)\right\|_{\infty}+c_{1}\left\|Q_{m, \bar{\xi}} h_{1}(y ; \cdot)\right\|_{\infty}\|\hat{\alpha}-\bar{\alpha}\|+ \\
& +B\left[\left\|\Gamma_{\hat{\xi}}-\Gamma_{\bar{\xi}}\right\|+\sigma^{-1}\left\|\Gamma_{\hat{\xi}}^{\prime}-\Gamma_{\bar{\xi}}^{\prime}\right\|\right] \leq \\
& \leq\left[B L+c_{1} A_{2}\right]\left[\left|\nu_{i}\right|+\delta_{i} \sqrt{\epsilon}\|y\|_{Y}\right]\|\hat{\alpha}-\bar{\alpha}\|+B\left[\left\|\Gamma_{\hat{\xi}}-\Gamma_{\bar{\xi}}\right\|+\sigma^{-1}\left\|\Gamma_{\hat{\xi}}^{\prime}-\Gamma_{\bar{\xi}}^{\prime}\right\|\right] .
\end{aligned}
$$

Now, if $e_{k}=e_{k+1}=1$ we have:

$$
\left|\Gamma_{\hat{\xi}, k}-\Gamma_{\bar{\xi}, k}\right| \leq \int_{\bar{\alpha}_{k}}^{\hat{\alpha}_{k}}|\dot{\gamma}(m-\theta)|+|\dot{\gamma}(-m-\theta)| d \theta \leq 2 \sigma A_{1} e^{2 \sigma} e^{-m \sigma}\left|\hat{\alpha}_{k}-\bar{\alpha}_{k}\right|
$$

and similarly

$\left|\Gamma_{\hat{\xi}, k}^{\prime}-\Gamma_{\bar{\xi}, k}^{\prime}\right| \leq \int_{\bar{\alpha}_{k}}^{\hat{\alpha}_{k}}|\ddot{\gamma}(m-\theta)|+|\ddot{\gamma}(-m-\theta)| d \theta \leq 2\left(\sigma^{2}+k N\right) A_{1} e^{2 \sigma} e^{-m \sigma}\left|\hat{\alpha}_{k}-\bar{\alpha}_{k}\right|$

Since similar estimates hold also in the other cases (i.e. when $\left(e_{k}, e_{k+1}\right)=(0,1)$, or $\left(e_{k}, e_{k+1}\right)=(1,0)$ or $\left.e_{k}=e_{k+1}=0\right)$ we obtain

$$
\begin{array}{r}
\left\|\Gamma_{\hat{\xi}}-\Gamma_{\bar{\xi}}\right\| \leq 2 \sigma A_{1} e^{2 \sigma} e^{-m \sigma}\|\hat{\alpha}-\bar{\alpha}\| \\
\left\|\Gamma_{\hat{\xi}}^{\prime}-\Gamma_{\bar{\xi}}^{\prime}\right\| \leq 2\left(\sigma^{2}+k N\right) A_{1} e^{2 \sigma} e^{-m \sigma}\|\hat{\alpha}-\bar{\alpha}\| .
\end{array}
$$

Hence using (38), (39) and the triangular inequality, the second estimate in (37) follows with

$$
\begin{aligned}
& \hat{k}_{1}=B L+A_{2} c_{1} \\
& \hat{k}_{2}=2 A_{1} B e^{2 \sigma}\left(2 \sigma+\sigma^{-1} k N\right) .
\end{aligned}
$$


This concludes the proof.

From Lemma 3 it follows that $T_{a}^{2}$ is a contraction on $Y_{\rho}$ provided $\epsilon$ and $\nu$ are sufficiently small. We now prove a similar result for $T_{b}^{2}$.

4. Lemma. There exist $\rho_{0}>0$ and a continuous increasing function $\widetilde{\Delta}(\rho)$ defined for $0 \leq \rho \leq \rho_{0}$ and such that $\widetilde{\Delta}(0)=0$, such that, for any $0<\rho<\rho_{0}$ the following hold

$$
\begin{aligned}
& \left\|T_{b}^{2}(\xi ; u)\right\|_{Y} \leq \frac{1}{2} A_{2} B \max \left\{k_{1}, k_{2}, N^{\prime}\right\}\|u\|^{2} \\
& \left\|T_{b}^{2}(\hat{\xi} ; \hat{u})-T_{b}^{2}(\bar{\xi} ; \bar{u})\right\|_{Y} \leq 3 A_{2} B \max \left\{k_{1}, k_{2}, N^{\prime}\right\} \rho\|\hat{u}-\bar{u}\|_{U} \\
& \quad+2 A_{2} B \rho \widetilde{\Delta}(\rho)\|\hat{\alpha}-\bar{\alpha}\|
\end{aligned}
$$

for any $u=(\phi, y, z), \hat{u}=(\hat{\phi}, \hat{y}, \hat{z}), \bar{u}=(\bar{\phi}, \bar{y}, \bar{z}) \in U_{\rho}$ and any $\hat{\xi}, \bar{\xi} \in X$ with $\hat{\xi}=(E, \hat{\alpha}), \bar{\xi}=(E, \bar{\alpha})$.

Proof. We begin with an estimate of $\left\|h_{2}(\xi, u ; \cdot)\right\|_{\infty}$. Using the second equality in (34) we get:

$$
\begin{aligned}
&\left|h_{2}(\xi, u ; t)\right| \leq\left|F\left(\gamma_{\xi}(t)+y(t), c(t)\right)-F\left(\gamma_{\xi}(t), 0\right)-F_{\eta}\left(\gamma_{\xi}(t), 0\right) y(t)\right| \\
&\left|\int_{0}^{1} F_{\eta}\left(\gamma_{\xi}(t)+\theta y(t), \theta c(t)\right)-F_{\eta}\left(\gamma_{\xi}(t), 0\right) d \theta y(t)\right| \\
&+\left|\int_{0}^{1} F_{\zeta}\left(\gamma_{\xi}(t)+\theta y(t), \theta c(t)\right) d \theta c(t)\right| .
\end{aligned}
$$

Now, from Lemma 1 we obtain

$$
\int_{0}^{1}\left|F_{\zeta}\left(\gamma_{\xi}(t)+\theta y(t), \theta c(t)\right)\right| d \theta|c(t)| \leq N^{\prime} \int_{0}^{1} \theta d \theta|c(t)|^{2} \leq \frac{1}{2} N^{\prime}|c(t)|^{2} .
$$

Next we have, using again Lemma 1,

$$
\begin{aligned}
& \int_{0}^{1}\left|F_{\eta}\left(\gamma_{\xi}(t)+\theta y(t), \theta c(t)\right)-F_{\eta}\left(\gamma_{\xi}(t), 0\right)\right| d \theta|y(t)| \\
\leq & \int_{0}^{1} \limsup _{\lambda \rightarrow 0^{+}} \int_{0}^{1}\left|F_{\eta \eta}\left(\gamma_{\xi}(t)+\sigma \theta y(t), \sigma \theta c(t)+\lambda\right)\right| \theta d \sigma d \theta|y(t)|^{2} \\
+ & \int_{0}^{1} \limsup _{\lambda \rightarrow 0^{+}} \int_{0}^{1}\left|F_{\eta \zeta}\left(\gamma_{\xi}(t)+\sigma \theta y(t), \sigma \theta c(t)+\lambda\right)\right| \theta d \sigma d \theta|c(t)||y(t)| \\
\leq & \frac{1}{2} k_{2}|y(t)|^{2}+\frac{1}{2} k_{3}|y(t)||c(t)| \leq \frac{1}{2} \max \left\{k_{2}, k_{3}\right\}|y(t)|(|y(t)|+|c(t)|)
\end{aligned}
$$

and then, using (36):

$$
\left\|h_{2}(\xi, u ; \cdot)\right\|_{\infty} \leq \frac{1}{2} \max \left\{k_{2}, k_{3}, N^{\prime}\right\}\left(\|\phi\|_{\Phi}+\|y\|_{Y}+\|z\|_{Z}\right)^{2} .
$$

Then the first estimate in (40) follows from $\left\|V_{m, \xi}\right\| \leq B$ and $\left\|Q_{m, \xi}\right\| \leq A_{2}$. 
Next, we observe that $\sqrt{\sum_{k=1}^{\infty} k^{2} a_{k}^{2}}$ defines a norm in the set of sequences $\left\{a_{k}\right\}_{k}$ such that $\sum_{k=1}^{\infty} k^{2} a_{k}^{2}<\infty$. Therefore, for any fixed $t$, the triangular inequality gives

$$
\begin{aligned}
i|\hat{c}(t)-\bar{c}(t)| & \leq\left|\left[\sum_{l=1}^{i-1} l^{2} \hat{\phi}_{l}^{2}(t)+\sum_{j=i+1}^{\infty} j^{2} \hat{z}_{j}^{2}(t)\right]^{1 / 2}-\left[\sum_{l=1}^{i-1} l^{2} \bar{\phi}_{l}^{2}(t)+\sum_{j=i+1}^{\infty} j^{2} \bar{z}_{j}^{2}(t)^{2}\right]^{1 / 2}\right| \\
& \leq\left\lfloor\sum_{l=1}^{i-1} l^{2}\left|\hat{\phi}_{l}(t)-\bar{\phi}_{l}(t)\right|^{2}+\sum_{j=i+1}^{\infty} j^{2}\left|\hat{z}_{j}(t)-\bar{z}_{j}(t)\right|^{2}\right]^{1 / 2}
\end{aligned}
$$

(here $\hat{c}_{j}(t)$ and $\bar{c}_{j}(t)$ are defined as in (35) with $\hat{\phi}_{l}, \bar{\phi}_{l}$ instead of $\phi_{l}$ etc.). Hence

$$
\|\hat{c}-\bar{c}\|_{\infty}^{2} \leq\|\hat{\phi}-\bar{\phi}\|_{\Phi}^{2}+i^{-4}\|\hat{z}-\bar{z}\|_{Z}^{2} .
$$

Now we prove the second estimate in (40). First we take $\hat{u}, \bar{u}, \in U_{\rho}$ with the same $y$ component, that is $\hat{u}=(\hat{\phi}, y, \hat{z}), \bar{u}=(\bar{\phi}, y, \bar{z})$. Then, using also (42) we get

$$
\begin{aligned}
& \left|h_{2}(\xi, \hat{\phi}, y, \hat{z} ; t)-h_{2}(\xi, \bar{\phi}, y, \bar{z} ; t)\right| \leq \\
& =\left|F\left(\gamma_{\xi}(t)+y(t), \hat{c}(t)\right)-F\left(\gamma_{\xi}(t)+y(t), \bar{c}(t)\right)\right| \leq \\
& \leq N^{\prime} \max \left\{\|\hat{c}\|_{\infty},\|\bar{c}\|_{\infty}\right\}\|\hat{c}-\bar{c}\|_{\infty}
\end{aligned}
$$

Thus using also (36) and (42) we obtain, for any $(\hat{\phi}, y, \hat{z}),(\bar{\phi}, y, \bar{z}) \in U_{\rho}$,

$$
\left\|h_{2}(\xi, \hat{\phi}, y, \hat{z} ; \cdot)-h_{2}(\xi, \bar{\phi}, y, \bar{z} ; \cdot)\right\|_{\infty} \leq N^{\prime} \rho \sqrt{2}\left[\|\hat{\phi}-\bar{\phi}\|_{\Phi}+i^{-2}\|\hat{z}-\bar{z}\|_{Z}\right] .
$$

Next we fix $\phi \in \Phi_{\rho}, z \in Z_{\rho}$ and take $\hat{y}, \bar{y} \in Y_{\rho}$. We have:

$$
\begin{aligned}
& \mid h_{2}(\xi, \phi, \hat{y}, z ; t)-h_{2}(\xi, \phi, \bar{y}, z ; t \mid \\
\leq & \int_{0}^{1}\left|F_{\eta}\left(\gamma_{\xi}+\theta \hat{y}+(1-\theta) \bar{y}, c\right)-F_{\eta}\left(\gamma_{\xi}, 0\right)\right| d \theta|\hat{y}(t)-\bar{y}(t)| \\
\leq & \left\{\int_{0}^{1} \limsup _{\lambda \rightarrow 0^{+}} \int_{0}^{1}\left|F_{\eta \eta}\left(\gamma_{\xi}+\sigma \theta \hat{y}+\sigma(1-\theta) \bar{y}, \sigma c+\lambda\right)\right||\theta \hat{y}+(1-\theta) \bar{y}| d \sigma d \theta\right. \\
& \left.+\int_{0}^{1} \limsup _{\lambda \rightarrow 0^{+}} \int_{0}^{1}\left|F_{\eta \zeta}\left(\gamma_{\xi}+\sigma \theta \hat{y}+\sigma(1-\theta) \bar{y}, \sigma c+\lambda\right)\right||c| d \sigma d \theta\right\}|\hat{y}(t)-\bar{y}(t)| \\
\leq & \left\{k_{2} \max \{|\hat{y}(t)|,|\bar{y}(t)|\}+k_{3}|c(t)|\right\}|\hat{y}(t)-\bar{y}(t)| \leq 3 \rho \max \left\{k_{2}, k_{3}\right\}|\hat{y}(t)-\bar{y}(t)|
\end{aligned}
$$

having also used (36). As a consequence for any $(\phi, \bar{y}, z),(\phi, \hat{y}, z) \in U_{\rho}$, we have:

$$
\left.\| h_{2}(\xi, \phi, \hat{y}, z ; \cdot)-h_{2}(\xi, \phi, \bar{y}, z), \cdot\right)\left\|_{\infty} \leq 3 \max \left\{k_{2}, k_{3}\right\} \rho\right\| \hat{y}-\hat{y} \|_{\infty}
$$

Now putting together the estimates (43), (44) and using $\left\|V_{m, \xi}\right\| \leq B$ and $\left\|Q_{m, \xi}\right\| \leq$ $A_{2}$ we see that

$$
\left\|T_{b}^{2}(\xi, \hat{u})-T_{b}^{2}(\xi, \bar{u})\right\|_{Y} \leq 3 A_{2} B \max \left\{k_{2}, k_{3}, N^{\prime}\right\} \rho\|\hat{u}-\bar{u}\|_{U}
$$

where $\hat{u}=(\hat{\phi}, \hat{y}, \hat{z}), \bar{u}=(\bar{\phi}, \bar{y}, \bar{z}) \in U_{\rho}$. This proves (40) when $\hat{\xi}=\bar{\xi}=\xi$.

Now we prove (40) when $\hat{u}=\bar{u}=u \in U_{\rho}$ and $\hat{\xi}=(E, \hat{\alpha}), \bar{\xi}=(E, \bar{\alpha})$, with $\hat{\alpha}, \bar{\alpha} \in \ell_{E}^{\infty}$. We have:

$$
\begin{aligned}
h_{2}(\hat{\xi}, u ; t) & -h_{2}(\bar{\xi}, u ; t)=F\left(\gamma_{\hat{\xi}}(t)+y(t), c(t)\right)-F\left(\gamma_{\bar{\xi}}(t)+y(t), c(t)\right) \\
& -F\left(\gamma_{\hat{\xi}}(t), 0\right)+F\left(\gamma_{\bar{\xi}}(t), 0\right)-\left[F_{\eta}\left(\gamma_{\hat{\xi}}(t), 0\right)-F\left(\gamma_{\bar{\xi}}(t), 0\right)\right] y(t) .
\end{aligned}
$$


Suppose that $t \in((2 j-1) m,(2 j+1) m]$. Then, if $e_{j}=0$ we have

$$
h_{2}(\hat{\xi}, u ; t)-h_{2}(\bar{\xi}, u ; t)=0
$$

since, in this case, $\gamma_{\hat{\xi}}(t)=\gamma_{\bar{\xi}}(t)=0$. If, instead, $e_{j}=1$ we have $\gamma_{\hat{\xi}}(t)=\gamma(t-2 j m-$ $\left.\hat{\alpha}_{j}\right)$ and similarly $\gamma_{\bar{\xi}}(t)=\gamma\left(t-2 j m-\bar{\alpha}_{j}\right)$. Assume, to fix ideas, that $\bar{\alpha}_{j} \geq \hat{\alpha}_{j}$ and set $I_{j}(t):=\left[t-2 j m-\bar{\alpha}_{j}, t-2 j m-\hat{\alpha}_{j}\right]$. Then we have, neglecting for simplicity dependence on $t$ in $y, c$ :

$$
\begin{aligned}
& \left|h_{2}(\hat{\xi}, u ; t)-h_{2}(\bar{\xi}, u ; t)\right| \leq \\
& \quad \limsup _{\lambda \rightarrow 0^{+}} \int_{I_{j}(t)} \int_{0}^{1}\left\{\left|F_{\eta \eta}(\gamma(s)+\theta y, \theta c+\lambda)-F_{\eta \eta}(\gamma(s), \lambda)\right||y|\right. \\
& \left.\quad+\left|F_{\eta \zeta}(\gamma(s)+\theta y, \theta c+\lambda) c\right|\right\}|\dot{\gamma}(s)| d \theta d s
\end{aligned}
$$

We have to distinguish two cases.

First case. If $\gamma(s) \geq 3 \rho$ then from (13) and $\sigma \leq 1$ we get, for any $\theta \in[0,1]$ :

$$
|\dot{\gamma}(s)| \leq \gamma(s)<2(\gamma(s)-\rho) \leq 2(\gamma(s)+\theta y) .
$$

Write, for simplicity, $a(s)=\gamma(s)+\theta y, b=\theta c+\lambda$ and note that $a(s) \geq 2 \rho$, $0 \leq b \leq \rho \sqrt{2}+\lambda$. Then

$$
\begin{aligned}
& \left|\dot{\gamma}(s) F_{\eta \zeta}(a(s), b)\right| \leq 2\left|a(s) F_{\eta \zeta}(a(s), b)\right| \\
& =2 b\left|4 a(s)^{3} f^{\prime \prime}\left(a(s)^{2}+b^{2}\right)+2 a(s) f^{\prime}\left(a(s)^{2}+b^{2}\right)\right| \leq \\
& \leq 2 b\left(N^{\prime}+4 N^{\prime \prime}\right) \leq 2\left(N^{\prime}+4 N^{\prime \prime}\right)(\rho \sqrt{2}+\lambda) .
\end{aligned}
$$

Next:

$$
\eta F_{\eta \eta}(\eta, \zeta)=6 \eta^{2} f^{\prime}\left(\eta^{2}+\zeta^{2}\right)+4 \eta^{4} f^{\prime \prime}\left(\eta^{2}+\zeta^{2}\right)
$$

is continuous in $\mathbb{R}^{2}$, and hence uniformly continuous in compact subsets of $\mathbb{R}^{2}$, since so are $x f^{\prime}(x)$ and $x^{2} f^{\prime \prime}(x)$ (see Remark 1-v). A a consequence, for $\left|\eta_{0}\right| \leq M$, $\left|\zeta_{0}\right| \leq 1$ and $\left|\eta-\eta_{0}\right|,\left|\zeta-\zeta_{0}\right| \leq \rho$ we have:

$$
\begin{aligned}
\left|\eta F_{\eta \eta}(\eta, \zeta)-\eta F_{\eta \eta}\left(\eta_{0}, \zeta_{0}\right)\right| & \leq\left|\eta F_{\eta \eta}(\eta, \zeta)-\eta_{0} F_{\eta \eta}\left(\eta_{0}, \zeta_{0}\right)\right|+\left|F_{\eta \eta}\left(\eta_{0}, \zeta_{0}\right)\right|\left|\eta-\eta_{0}\right| \\
& \leq \Delta\left(\left|\eta-\eta_{0}\right|+\left|\zeta-\zeta_{0}\right|\right)+k_{2}\left|\eta-\eta_{0}\right|
\end{aligned}
$$

where $\Delta(\rho)$ is an increasing function of $\rho$ such that $\Delta(\rho) \rightarrow 0$ as $\rho \rightarrow 0$. Taking

$$
\eta_{0}=\gamma(s), \quad \zeta_{0}=\lambda, \quad \eta=\gamma(s)+\theta y, \quad \zeta=\theta c+\lambda
$$

we see that:

$$
\left|F_{\eta \eta}(\gamma(s)+\theta y, \theta c+\lambda)-F_{\eta \eta}(\gamma(s), \lambda)\right||\dot{\gamma}(s)| \leq 2 \Delta(|y|+|c|)+2 k_{2}|y|
$$

Second case. If $\gamma(s)<3 \rho$ then $|\dot{\gamma}(s)| \leq \gamma(s)<3 \rho$ and

$$
\left|F_{\eta \zeta}(\gamma(s)+\theta y, \theta c+\lambda) c\right| \dot{\gamma}(s) \leq 6 k_{3} \rho^{2}
$$

and

$$
\left.\mid\left[F_{\eta \eta}(\gamma(s)+\theta y, \theta c+\lambda)-F_{\eta \eta}(\gamma(s), \lambda)\right] y \dot{\gamma}(s)\right) \mid \leq 6 k_{2} \rho^{2}
$$

Summarizing, from (46), (47)-(50), we get

$$
\left|h_{2}(\hat{\xi}, u ; t)-h_{2}(\bar{\xi}, u ; t)\right| \leq 2 \rho\left\{\Delta(\|u\|)+2\left(N^{\prime}+4 N^{\prime \prime}\right) \rho+3\left(k_{2}+k_{3}\right) \rho\right\}\left|\hat{\alpha}_{j}-\bar{\alpha}_{j}\right|
$$

and hence

$$
\left\|h_{2}(\hat{\xi}, u ; \cdot)-h_{2}(\bar{\xi}, u ; \cdot)\right\|_{\infty} \leq 2 \rho \widetilde{\Delta}(\rho)\|\hat{\alpha}-\bar{\alpha}\|
$$


where $\widetilde{\Delta}(\rho)=\Delta(3 \rho)+2\left(N^{\prime}+4 N^{\prime \prime}\right) \rho+3\left(k_{2}+k_{3}\right) \rho$. Thus (40) with $\hat{u}=\bar{u}$ follows from $\left\|V_{m, \xi}\right\| \leq B$ and $\left\|Q_{m, \xi}\right\| \leq A_{2}$. The second estimate in (40) follows now easily from (45) and (51). The proof is complete.

Note that the second estimate in (40) implies that $T_{b}^{2}$ is a contraction with respect to the $u$ variable (provided $3 A_{2} B \max \left\{k_{1}, k_{2}, N^{\prime}\right\} \rho<1$ ) with factor of contraction $3 A_{2} B \max \left\{k_{1}, k_{2}, N^{\prime}\right\} \rho$. Thus putting the results of Lemmas 3 and 4 together we find the following.

2. Proposition. Consider the operator $T^{2}: U_{\rho} \times X \times \mathbb{N}_{m_{0}} \times \mathbb{R}_{+} \rightarrow Y$. There are constants $k_{4}>0$, and $\rho_{0}>0$ so that, for any $0<\rho<\rho_{0}, \nu \in \mathbb{R}, m>m_{0}$ and $\epsilon>0$, we have the following:

$$
\left\|T^{2}(u, \xi, m, \epsilon)\right\|_{Y} \leq k_{4}\left[\left|\nu_{i}\right|+e^{-m \sigma}\right]+A_{2} B\left\{\max \left\{k_{1}, k_{2}, N^{\prime}\right\} \frac{\|u\|_{U}}{2}+\sqrt{\epsilon} \delta_{i}\right\}\|u\|_{U} .
$$

Therefore if $0<\rho<\rho_{0}$ and $0<\epsilon<\epsilon_{0}$ where $\rho_{0}$ and $\epsilon_{0}$ satisfy:

$$
3 A_{2} B\left[\max \left\{k_{1}, k_{2}, N^{\prime}\right\} \rho_{0}+2 \sqrt{\epsilon_{0}} \delta_{i}\right]<2
$$

and $|\nu|<\bar{\nu}(\rho), \bar{m}(\rho)<m \in \mathbb{N}$ are chosen so that

$$
2 k_{4}\left[i^{-1} \bar{\nu}(\rho)+e^{-\bar{m}(\rho) \sigma}\right]<\rho,
$$

then $T^{2}$ maps $U_{\rho}$ in $Y_{\rho}$. Moreover if $\hat{u}:=(\hat{\phi}, \hat{y}, \hat{z}), \bar{u}:=(\bar{\phi}, \bar{y}, \bar{z}) \in U_{\rho}$ and $\hat{\xi}=$ $(E, \hat{\alpha}), \bar{\xi}=(E, \bar{\alpha}) \in X$, we have

$$
\begin{aligned}
& \left\|T^{2}(\hat{u}, \hat{\xi}, m, \epsilon)-T^{2}(\bar{u}, \bar{\xi}, m, \epsilon)\right\|_{Y} \leq 3 A_{2} B\left[\max \left\{k_{1}, k_{2}, N^{\prime}\right\} \rho+\sqrt{\epsilon} \delta_{i}\right]\|\hat{u}-\bar{u}\|_{U} \\
+ & {\left[\hat{k}_{1}\left(\left|\nu_{i}\right|+\sqrt{\epsilon} \delta_{i} \rho\right)+\hat{k}_{2} e^{-m \sigma}+2 A_{2} B \widetilde{\Delta}(\rho) \rho\right]\|\hat{\alpha}-\bar{\alpha}\| . }
\end{aligned}
$$

In analogy to what we have done for the $y$-component, we construct an operator $T^{1}$, whose fixed points are the bounded solutions of equation (5). This corresponds to looking for the unique bounded solution in the $i-1$ dimensional hyperbolic manifold. Observe that, when the load $\gamma$ is close to the first eigenvalue of the unperturbed problem (that is $i=1$ ), the hyperbolic manifold reduce to a point: the origin. However when $i>1$ the hyperbolic manifold is not trivial; it follows that, in these cases, the chaotic phenomenon which will be described below is highly unstable, even if it may influence the actual dynamics of the real phenomena. Also observe that the number $i$ equals the number of humps that can be observed in the beam (spatially), when the solution is close to the unperturbed homoclinic.

Consider the linear inhomogeneous problem associated to (8):

$$
\ddot{\phi}_{l}(t)+\sqrt{\epsilon} \delta_{i} \dot{\phi}_{l}(t)-\frac{l^{2}}{i^{2}} \frac{i^{2}-l^{2}+\epsilon \sigma^{2}}{\epsilon} \phi_{l}(t)=h_{l}(t),
$$

where $0<l<i$. Note that this part is needed only if $i \geq 2$ and then $i^{2}-l^{2} \geq 3$. We set $X_{l}(t)=\left(\begin{array}{c}\phi_{l}(t) \\ \dot{\phi}_{l}(t)\end{array}\right)$ and rewrite $(53)$ as

$$
\dot{X}_{l}(t)=A_{l} X_{l}(t)+b_{l}(t),
$$

where

$$
A_{l}:=\left(\begin{array}{cc}
0 & 1 \\
\frac{l^{2}}{i^{2}} \frac{i^{2}-l^{2}+\epsilon \sigma^{2}}{\epsilon} & -\sqrt{\epsilon} \delta_{i}
\end{array}\right) \quad b_{l}(t)=\left(\begin{array}{c}
0 \\
h_{l}(t)
\end{array}\right) .
$$


The eigenvalues of $A_{l}$ are

$$
\lambda_{ \pm}=\frac{1}{2 i \sqrt{\epsilon}}\left(-\delta \epsilon \pm \sqrt{\delta^{2} \epsilon^{2}+4 l^{2}\left(i^{2}-l^{2}+\epsilon \sigma^{2}\right)}\right)
$$

and hence are real and have opposite sign. Moreover $0<\lambda_{+} \leq\left|\lambda_{-}\right|$and

$$
\left|\lambda_{ \pm}\right| \geq \iota:=\frac{1}{i \sqrt{\epsilon}}
$$

provided $\delta \epsilon<i^{2}-2$, since $\sqrt{\delta^{2} \epsilon^{2}+4 l^{2}\left(i^{2}-l^{2}+\epsilon \sigma^{2}\right)} \geq \sqrt{\delta^{2} \epsilon^{2}+4 l^{2}\left(i^{2}-l^{2}\right)}>$ $2+\delta \epsilon$.

As a consequence equation (54) with $b_{l}(t)=0$ admits an exponential dichotomy on $\mathbb{R}$ with projection

$$
\mathbf{P}=\frac{1}{\lambda_{+}-\lambda_{-}}\left(\begin{array}{cc}
\lambda_{+} & -1 \\
\lambda_{-} \lambda_{+} & -\lambda_{-}
\end{array}\right)
$$

and exponent $\iota$, if $\epsilon \delta<i^{2}-2$. However the constants of the dichotomy depend on $\epsilon$. To study this dependence we observe the following. The eigenvectors of $\lambda_{ \pm}$are, respectively:

$$
v_{+}=\left(\begin{array}{c}
1 \\
\lambda_{+}
\end{array}\right) \quad v_{-}=\left(\begin{array}{c}
1 \\
\lambda_{-}
\end{array}\right)
$$

( $v_{+}$spans the unstable space and $v_{-}$the stable space). Let

$$
V=\left(\begin{array}{cc}
1 & 1 \\
\lambda_{+} & \lambda_{-}
\end{array}\right)
$$

Then the fundamental matrix $e^{A_{l} t}$ of (54) is

$$
e^{A_{l} t}=V\left(\begin{array}{cc}
e^{\lambda_{+} t} & 0 \\
0 & e^{\lambda_{-} t}
\end{array}\right) V^{-1}
$$

from which we obtain:

$$
e^{A_{l} t} \mathbf{P}=e^{\lambda_{-} t} \mathbf{P}, \quad e^{A_{l} t}(\mathbf{I}-\mathbf{P})=e^{\lambda_{+} t}(\mathbf{I}-\mathbf{P}) .
$$

Next, since $i \sqrt{\epsilon}\left(\lambda_{+}-\lambda_{-}\right)=\sqrt{\delta^{2} \epsilon^{2}+4 l^{2}\left(i^{2}-l^{2}+\epsilon \sigma^{2}\right)}, i\left(\lambda_{+}+\lambda_{-}\right)=-\sqrt{\epsilon} \delta$, and $i^{2} \lambda_{+} \lambda_{-}=-l^{2}\left(i^{2}-l^{2}+\epsilon \sigma^{2}\right) \epsilon^{-1}$, we have

$$
A_{l}=\left(\begin{array}{cc}
0 & 1 \\
-\lambda_{+} \lambda_{-} & \lambda_{+}+\lambda_{-}
\end{array}\right) .
$$

Hence it is easily checked that $A_{l} \mathbf{P}=\mathbf{P} A_{l}$ and $e^{A_{l} t} \mathbf{P} e^{-A_{l} s}=e^{A_{l}(t-s)} \mathbf{P}$. So:

$$
\begin{array}{cc}
\left\|e^{A_{l} t} \mathbf{P} e^{-A_{l} s}\right\| \leq\|\mathbf{P}\| e^{\lambda_{-}(t-s)} \leq\|\mathbf{P}\| e^{-\iota(t-s)}, & \text { for } s \leq t \\
\left\|e^{A_{l} t}(\mathbf{I}-\mathbf{P}) e^{-A_{l} s}\right\| \leq\|\mathbf{I}-\mathbf{P}\| e^{\lambda_{+}(t-s)} \leq\|\mathbf{I}-\mathbf{P}\| e^{-\iota(s-t)}, & \text { for } t \leq s .
\end{array}
$$

Finally, it is a simple computation to verify that

$$
\|\mathbf{I}-\mathbf{P}\|^{2}=\|\mathbf{P}\|^{2}=\frac{\left(\lambda_{+}^{2}+1\right)\left(\lambda_{-}^{2}+1\right)}{\left(\lambda_{+}-\lambda_{-}\right)^{2}}=\frac{1}{4 \epsilon} \frac{16 \ell^{4}\left(i^{2}-\ell^{2}\right)^{2}+o(1)}{4 \ell^{2}\left(i^{2}-\ell^{2}\right)+o(1)}
$$

where $o(1) \rightarrow 0$ as $\epsilon \rightarrow 0$. Thus there are $\epsilon_{0}>0$ and $K=\frac{1}{2} \sqrt{i^{4}+4}>1$ such that for $0<\epsilon<\epsilon_{0}$ it results

$$
\|\mathbf{I}-\mathbf{P}\|=\|\mathbf{P}\| \leq K \epsilon^{-1 / 2} .
$$


As a consequence (54) has an exponential dichotomy on $\mathbb{R}$ with projection $\mathbf{P}$, exponent $\frac{1}{i \sqrt{\epsilon}}$ and constant $K \epsilon^{-1 / 2}$. Hence, for any bounded function $h_{l}(t) \in$ $L^{\infty}(\mathbb{R})$, system (54) has the unique bounded (weak) solution:

$$
\begin{aligned}
\hat{X}_{l}(t)=\left(\begin{array}{c}
\hat{\phi}_{l}(t) \\
\dot{\hat{\phi}}_{l}(t)
\end{array}\right):=\int_{-\infty}^{t} e^{A_{l}(t-s)} \mathbf{P}\left(\begin{array}{c}
0 \\
h_{l}(s)
\end{array}\right) d s \\
-\int_{t}^{+\infty} e^{A_{l}(t-s)}(\mathbf{I}-\mathbf{P})\left(\begin{array}{c}
0 \\
h_{l}(s)
\end{array}\right) d s \\
=\int_{-\infty}^{t} e^{\lambda_{-}(t-s)} \mathbf{P}\left(\begin{array}{c}
0 \\
h_{l}(s)
\end{array}\right) d s-\int_{t}^{+\infty} e^{\lambda_{+}(t-s)}(\mathbf{I}-\mathbf{P})\left(\begin{array}{c}
0 \\
h_{l}(s)
\end{array}\right) d s
\end{aligned}
$$

We construct the linear operator $L_{l}^{\phi}: L^{\infty}(\mathbb{R}) \rightarrow C_{b}^{1}(\mathbb{R})$ that associates to $h_{l}(t)$ the function $\hat{\phi}_{l}(t) \in C_{b}^{1}(\mathbb{R})$ defined by $(55)$. Then we define the operator $T_{a}^{1}(u, \xi, m, \epsilon)$ : $U_{\rho} \times X \times \mathbb{N}_{m_{0}} \times \mathbb{R}_{+} \rightarrow \Phi$ and $T_{b}^{1}(h, m, \epsilon): L^{\infty}(\mathbb{R}) \times \mathbb{N}_{m_{0}} \times \mathbb{R}_{+} \rightarrow \Phi$ as follows:

$$
T_{a}^{1}(u, \xi, m, \epsilon)=\left\{\Phi_{l}^{a}\right\}_{l=1, \ldots, i-1}, \quad T_{b}^{1}(h, m, \epsilon)=\left\{\Phi_{l}^{b}\right\}_{l=1, \ldots, i-1},
$$

where

$$
\Phi_{l}^{a}:=-L_{l}^{\phi}\left[k \mathfrak{F}(\xi, u, t) \frac{l^{2}}{i^{2}} \phi_{l}\right], \quad \Phi_{l}^{b}:=L_{l}^{\phi}\left[\nu_{i} \int_{0}^{\pi} h(x, t / i) \sqrt{\frac{2}{\pi}} \sin (l x) \mathrm{d} x\right]
$$

Finally we introduce the operator $T^{1}(u, \xi, m, \epsilon)=T_{a}^{1}(u, \xi, m, \epsilon)+T_{b}^{1}(h, m, \epsilon)$ where $h$ is the forcing term of (1). We will need the following result.

5. Lemma. Consider a function $h \in L^{\infty}(\mathbb{R})$. Then $L_{l}^{\phi}(h) \in C_{b}^{1}(\mathbb{R})$ and

$$
\left\|L_{l}^{\phi}(h)\right\|_{C_{b}^{1}} \leq 2 i \sqrt{\epsilon}\|h\|_{\infty}
$$

provided $\epsilon>0$ is sufficiently small.

Proof. The fact that $L_{l}^{\phi}(h) \in C_{b}^{1}(\mathbb{R})$ is a trivial consequence of the definition of the operator. To show (57) we first observe that

$$
\lim _{\epsilon \rightarrow 0} \frac{1+\lambda_{-}^{2}}{\left(\lambda_{+}-\lambda_{-}\right)^{2}}=\lim _{\epsilon \rightarrow 0} \frac{1+\lambda_{+}^{2}}{\left(\lambda_{+}-\lambda_{-}\right)^{2}}=\frac{1}{4} .
$$

Then we get

$$
\left\|\mathbf{P}\left(\begin{array}{c}
0 \\
h
\end{array}\right)\right\|=\left\|\frac{1}{\lambda_{-}-\lambda_{+}}\left(\begin{array}{c}
1 \\
\lambda_{-}
\end{array}\right) h\right\| \leq\|h\|_{\infty}
$$

provided $\epsilon$ is sufficiently small. Similarly,

$$
\left\|(\mathbf{I}-\mathbf{P})\left(\begin{array}{c}
0 \\
h
\end{array}\right)\right\|=\left\|\frac{1}{\lambda_{+}-\lambda_{-}}\left(\begin{array}{c}
1 \\
\lambda_{+}
\end{array}\right) h\right\| \leq\|h\|_{\infty} .
$$

Then, from (55) we get

$$
\begin{aligned}
\left|L_{l}^{\phi}(h)(t)\right| & \leq\left[\int_{0}^{+\infty} e^{\lambda_{-} s} d s+\int_{-\infty}^{0} e^{-\lambda_{+} s} d s\right]\|h\|_{\infty} \\
& \leq\left(\frac{1}{\lambda_{+}}-\frac{1}{\lambda_{-}}\right)\|h\|_{\infty} \leq 2 i \sqrt{\epsilon}\|h\|_{\infty}
\end{aligned}
$$

for $\epsilon>0$ sufficiently small, and (57) follows.

Now we are ready to state and prove the following result. 
3. Proposition. There exist positive numbers $\epsilon_{0}, \rho_{0}<1$ and $m_{0}>1$ such that if $0<\rho<\rho_{0}, 0<\epsilon<\epsilon_{0}$ and $m>m_{0}, u \in U_{\rho}$ we have

$$
\left\|T^{1}(u, \xi, m, \epsilon)\right\|_{\Phi} \leq 2 \sqrt{\epsilon}\left[\nu+k N i\left\|_{\phi}\right\|_{\Phi}\right] .
$$

Hence $T^{1}$ maps $\Phi_{\rho}$ into $\Phi_{\rho}$ provided $0<\epsilon<\epsilon_{0}$ where

$$
4 k N i \sqrt{\epsilon_{0}}<1 \text { and } 0<\nu<\nu(\rho)=k N i \rho .
$$

Furthermore $T^{1}$ is a contraction in the $u$ and $\xi$ variables. More precisely, for any $\hat{u}, \bar{u} \in U_{\rho}$ and any $\hat{\xi}, \bar{\xi} \in X$ we have

$$
\begin{aligned}
\| T^{1}(\hat{u}, \hat{\xi}, m, \epsilon) & -T^{1}(\bar{u}, \bar{\xi}, m, \epsilon) \|_{\Phi} \\
& \leq 2 k N^{\prime}(M+1) i \sqrt{i-1} \sqrt{\epsilon}\left[2\|\hat{u}-\bar{u}\|_{U}+\rho\|\hat{\alpha}-\bar{\alpha}\|\right]
\end{aligned}
$$

Proof. We recall that $T^{1}(u, \xi, m, \epsilon)=T_{a}^{1}(u, \xi, m, \epsilon)+T_{b}^{1}(h, m, \epsilon)$ where $T_{a}^{1}$ and $T_{b}^{1}$, have been defined in (56). We set, for simplicity

$$
c_{l}(t):=\left[i^{-2} \sum_{p=1, p \neq l}^{i-1} p^{2} \phi_{p}^{2}(t)+\left(\gamma_{\xi}(t)+y(t)\right)^{2}+i^{-2} \sum_{j>i} j^{2} z_{j}^{2}(t)\right]^{1 / 2} .
$$

Note that, for any $t \in \mathbb{R}$

$$
c_{l}(t)=\left\|\left(\begin{array}{c}
\left\{(p / i) \phi_{p}(t)\right\}_{l \neq p<i} \\
\gamma_{\xi}(t)+y(t) \\
\left\{(j / i) z_{j}(t)\right\}_{j>i}
\end{array}\right)\right\| .
$$

Then, since (see (29), (14)) $\mathfrak{F}(\xi, u, t) \frac{l}{i} \phi_{l}(t)=F\left(\frac{l}{i} \phi_{l}(t), c_{l}(t)\right),|F(\eta, \zeta)| \leq N|\eta|$ when $\eta^{2}+\zeta^{2} \leq M^{2}+1$, and $c_{l}(t)^{2}+\left(\frac{l}{i} \phi_{l}\right)^{2} \leq\|\phi\|_{\Phi}^{2}+\left(\left\|\gamma_{\xi}\right\|_{\infty}+\|y\|_{\infty}\right)^{2}+i^{-4}\|z\|_{Z}^{2} \leq$ $2 \rho^{2}+(M+\rho)^{2}$, we see that (if $0<\rho \leq\left(\sqrt{M^{2}+3}-M\right) / 3$ )

$$
\left\|\Phi_{l}^{a}\right\|_{C_{b}^{1}(\mathbb{R})} \leq 2 k i \sqrt{\epsilon}(l / i)^{2} N\left\|\phi_{l}\right\|_{C_{b}^{1}(\mathbb{R})}
$$

having used (57). As a consequence we obtain

$$
\left\|T_{a}^{1}(u, \xi, m, \epsilon)\right\|_{\Phi} \leq 2 k N i \sqrt{\epsilon}\|\phi\|_{\Phi}
$$

Similarly, using again Lemma 5 we get

$$
\left\|T_{b}^{1}(h, m, \epsilon)\right\|_{\Phi} \leq 2 \nu \sqrt{\epsilon} .
$$

Putting (61), (62) together we obtain (58) and

$$
\left\|T^{2}(u, \xi, m, \epsilon)\right\|_{\Phi}<\rho
$$

for any $\phi \in \Phi_{\rho}$ provided

$$
4 k N i \sqrt{\epsilon}<1 \text { and } \nu<\nu(\rho)=k N i \rho .
$$

Next we prove (59). Let $\hat{\phi}, \bar{\phi} \in \Phi_{\rho}, \hat{y}, \bar{y} \in Y_{\rho}, \hat{z}, \bar{z} \in Z_{\rho}$ and write $\hat{c}(t), \bar{c}(t)$ for the functions defined as in $(60)$ with $(\hat{\phi}, \hat{y}, \hat{z})$, resp. $(\bar{\phi}, \bar{y}, \bar{z})$, instead of $(\phi, y, z)$. Then we obtain using Lemma 1 :

$$
\begin{aligned}
& \left|F\left(\frac{l}{i} \hat{\phi}_{l}(t), \hat{c}_{l}(t)\right)-F\left(\frac{l}{i} \bar{\phi}_{l}(t), \bar{c}_{l}(t)\right)\right| \\
& \leq N^{\prime}\left(\rho+\sqrt{M^{2}+1}\right)\left[\frac{l}{i}\left|\hat{\phi}_{l}(t)-\bar{\phi}_{l}(t)\right|+\left|\hat{c}_{l}(t)-\bar{c}_{l}(t)\right|\right] \\
& \leq N^{\prime}\left(\rho+\sqrt{M^{2}+1}\right)\left[\sqrt{2}\|\hat{\phi}-\bar{\phi}\|_{\Phi}+\|\hat{y}-\bar{y}\|_{Y}+\|\hat{z}-\bar{z}\|_{Z}\right] \\
& \leq 2 N^{\prime}(M+1)\|\hat{u}-\bar{u}\|_{U},
\end{aligned}
$$

provided $0<\rho<\rho_{0}$ with $\rho_{0}>0$ is sufficiently small. 
Thus, since $T_{b}^{1}$ does not depend on $u$, we see that (59) is satisfied when $\hat{\xi}=\bar{\xi}$. Finally we prove (59) when $\hat{u}=\bar{u}$ and $\hat{\xi}, \bar{\xi}$ are possibly different. Again it is enough to prove it for the operator $T_{a}^{1}$, since $T_{b}^{1}$ does not depend on $\xi$. From Lemma 1 we get:

$$
\begin{aligned}
\left|F\left(\frac{l}{i} \phi_{l}(t), \hat{c}_{l}(t)\right)-F\left(\frac{l}{i} \phi_{l}(t), \bar{c}_{l}(t)\right)\right| & \leq N^{\prime} \frac{l}{i}\left|\phi_{l}(t)\right|\left|\hat{c}_{l}(t)-\bar{c}_{l}(t)\right| \\
\leq N^{\prime} \rho\left|\gamma_{\hat{\xi}}(t)-\gamma_{\bar{\xi}}(t)\right| & \leq N^{\prime} M \sigma \rho\|\hat{\alpha}-\bar{\alpha}\| \leq N^{\prime} M \rho\|\hat{\alpha}-\bar{\alpha}\|
\end{aligned}
$$

and then (59) with $\hat{u}=\bar{u}$ easily follows from Lemma 5 . This concludes the proof of the Proposition.

Now we study equation (32) that is the restriction of the original problem to the center manifold. Thus we construct an operator $T^{3}(u, \xi, m, \epsilon): U_{\rho} \times X \times \mathbb{N}_{m_{0}} \times$ $\mathbb{R}_{+} \rightarrow Z_{\rho}$ whose fixed points solve (32). We consider, first, the following linear problems associated to $(32)$ :

$$
\ddot{z}_{j}(t)+\sqrt{\epsilon} \delta_{i} \dot{z}_{j}(t)+\frac{j^{2}-i^{2}-\epsilon \sigma^{2}}{\epsilon} \frac{j^{2}}{i^{2}} z_{j}(t)=h_{j}(t), \quad j>i .
$$

Assume $\epsilon \delta_{i} \leq 2$ and $\epsilon \sigma^{2}<2$. Then the only bounded solutions of (63) is given by

$$
z_{j}(t)=L_{j}^{z}\left(h_{j}(t)\right):=\frac{2 \sqrt{\epsilon}}{\omega_{j, \epsilon}} \int_{-\infty}^{t} e^{-\frac{\sqrt{\epsilon} \delta_{i}(t-s)}{2}} \sin \left(\frac{\omega_{j, \epsilon}(t-s)}{2 \sqrt{\epsilon}}\right) \times h_{j}(s) d s
$$

where $\omega_{j, \epsilon}=\sqrt{4 \frac{j^{2}}{i^{2}}\left(j^{2}-i^{2}-\epsilon \sigma^{2}\right)-\epsilon^{2} \delta_{i}^{2}} \geq 2 \sqrt{\frac{j^{2}}{i^{2}}\left(j^{2}-i^{2}-2\right)-1} \geq \frac{1}{2} \frac{j^{2}}{i^{2}}$ and define the operator $T^{3}(u, \xi, m, \epsilon): U_{\rho} \times X \times \mathbb{N}_{m_{0}} \times \mathbb{R}_{+} \rightarrow Z$, as follows: $T^{3}(u, \xi, m, \epsilon)(t)=$ $\breve{z}(t)=\left\{\breve{z}_{j}(t)\right\}_{j>i}$ where

$$
\breve{z}_{j}(t):=L_{j}^{z}\left[-\frac{k j^{2}}{i^{2}} \mathfrak{F}(\xi, \phi, y, z, t) z_{j}+\nu_{i} \int_{0}^{\pi} h(x, t / i) \sqrt{\frac{2}{\pi}} \sin (j x) \mathrm{d} x\right]
$$

To give a good estimate of $T^{3}$, when $\gamma_{\xi}$ is periodic or anyway $\xi$ has an infinite subsequence of 1 we need the following technical Lemma.

6. Lemma. Let $g: \mathbb{R}_{+} \rightarrow \mathbb{R}_{+}$be a continuous increasing function such that $g(0)=0$ and $a \geq 0$ be $a$ non negative number. Then there exists $\epsilon_{0}>0$ such that for $0<\epsilon<\epsilon_{0}$ and for any $t \in \mathbb{R}$ and $j \in \mathbb{N}$ we have

$$
\begin{gathered}
\int_{0}^{\infty} e^{-\sqrt{\epsilon} \delta s} g\left(a \rho^{2}+\left(\gamma_{\xi}(t-s)+\rho\right)^{2}\right) d s \leq 4 \frac{g\left(a \rho^{2}+(M+\rho)^{2}\right)}{\epsilon^{1 / 4}\left(1-e^{-2 m \sqrt{\epsilon} \delta}\right)} \\
+\frac{g\left(a \rho^{2}+(\rho+\epsilon)^{2}\right)}{\sqrt{\epsilon} \delta} .
\end{gathered}
$$

Proof. For any $k \in \mathbb{Z}$ we set $t_{k}=t-2 k m$ and note that

$$
t_{k-1}-m=t_{k}+m \text {. }
$$

Then, for any fixed $t \in \mathbb{R}$ and $j \in \mathbb{N}$, there is a unique integer $\ell$ (i.e. $\ell=$ $\left.\left[\frac{t-(2 j+1) m}{2 m}\right]\right)$ such that $t-2(j+1) m \leq(2 \ell-1) m<t-2 j m$ or, in other words, $(2 \ell-1) m<t_{j} \leq(2 \ell+1) m$. Note that $\gamma_{\xi}\left(t_{j}-s\right)$ is a $C^{1}$-function of $s$ in any of the two intervals $\left[0, t_{j+\ell}+m\right]$ and $\left[t_{j+\ell}+m, 2 m\right]$ (here continuity at the end points means that the limits exist in $\mathbb{R}$ ). As a first step we estimate

$$
\int_{0}^{2 m} e^{-\sqrt{\epsilon} \delta s} g\left(a \rho^{2}+\left(\gamma_{\xi}\left(t_{j}-s\right)+\rho\right)^{2}\right) d s
$$


To this end we write $[0,2 m]=\left[0, t_{j+\ell}+m\right] \cup\left[t_{j+\ell}+m, 2 m\right]$ and split the intervals $\left[0, t_{j+\ell}+m\right],\left[t_{j+\ell}+m, 2 m\right]$ as:

$$
\begin{aligned}
& {\left[0, t_{j+\ell}+m\right]=I_{1}^{j} \cup I_{2}^{j} \cup I_{3}^{j}} \\
& {\left[t_{j+\ell}+m, 2 m\right]=J_{1}^{j} \cup J_{2}^{j} \cup J_{3}^{j}}
\end{aligned}
$$

where

$$
\begin{aligned}
I_{1}^{j} & =\left[0, t_{j+\ell}-\epsilon^{-1 / 4}\right], & I_{2}^{j} & =\left[t_{j+\ell}-\epsilon^{-1 / 4}, t_{j+\ell}+\epsilon^{-1 / 4}\right], \\
I_{3}^{j} & =\left[t_{j+\ell}+\epsilon^{-1 / 4}, t_{j+\ell}+m\right], & J_{1}^{j} & =\left[t_{j+\ell-1}-m, t_{j+\ell-1}-\epsilon^{-1 / 4}\right], \\
J_{2}^{j} & =\left[t_{j+\ell-1}-\epsilon^{-1 / 4}, t_{j+\ell-1}+\epsilon^{-1 / 4}\right], & J_{3}^{j} & =\left[t_{j+\ell-1}+\epsilon^{-1 / 4}, 2 m\right] .
\end{aligned}
$$

Note that we only need to consider four of the above intervals. For example $\left[0, t_{j+\ell}-\right.$ $\left.\epsilon^{-1 / 4}\right]$ needs to be considered only if $t_{j+\ell}>\epsilon^{-1 / 4}$. But in this case $t_{j+\ell-1}-\epsilon^{-1 / 4}>$ $2 m$ and then we will take $J_{2}^{j}=J_{3}^{j}=\emptyset$ and replace $J_{1}^{j}$ with $\left[t_{j+\ell-1}-m, 2 m\right]$. Similarly if $-\epsilon^{-1 / 4}<t_{j+\ell} \leq \epsilon^{-1 / 4}$ we can take $I_{1}^{j}=J_{3}^{j}=\emptyset, I_{2}^{j}=\left[0, t_{j+\ell}+\epsilon^{-1 / 4}\right]$ and $J_{2}^{j}=\left[t_{j+\ell-1}-\epsilon^{-1 / 4}, 2 m\right]$. Finally, if $t_{j+\ell}+\epsilon^{-1 / 4} \leq 0$ we can take $I_{1}^{j}=I_{2}^{j}=\emptyset$, $I_{3}^{j}=\left[0, t_{j+\ell}+m\right]$.

Next we observe that

$$
\begin{aligned}
& s \in I_{1}^{j} \Rightarrow 2 \ell m+\epsilon^{-1 / 4} \leq t_{j}-s \leq t_{j}<(2 \ell+1) m \\
& s \in I_{3}^{j} \Rightarrow(2 \ell-1) m \leq t_{j}-s \leq 2 \ell m-\epsilon^{-1 / 4} \\
& s \in J_{1}^{j} \Rightarrow 2(\ell-1) m+\epsilon^{-1 / 4} \leq t_{j}-s \leq(2 \ell-1) m \\
& s \in J_{3}^{j} \Rightarrow(2 \ell-3) m \leq t_{j}-s \leq 2(\ell-1) m-\epsilon^{-1 / 4} .
\end{aligned}
$$

Using (18) we see that:

$$
\sup _{s \in I_{1}^{j} \cup I_{3}^{j} \cup J_{1}^{j} \cup J_{3}^{j}}\left|\gamma_{\xi}\left(t_{j}-s\right)\right| \leq A_{1} e^{-\sigma\left(\epsilon^{-1 / 4}-2\right)} .
$$

Next,

$$
\operatorname{meas}\left(I_{2}^{j}\right) \leq 2 \epsilon^{-1 / 4}, \quad \operatorname{meas}\left(J_{2}^{j}\right) \leq 2 \epsilon^{-1 / 4}
$$

thus:

$$
\int_{\mathcal{I}_{2}^{j}} e^{-\sqrt{\epsilon} \delta s} g\left(a \rho^{2}+\left(\gamma_{\xi}\left(t_{j}-s\right)+\rho\right)^{2}\right) d s \leq 2 g\left(a \rho^{2}+(M+\rho)^{2}\right) \epsilon^{-1 / 4} .
$$

where $\mathcal{I}_{2}^{j}$ is either $I_{2}^{j}$ or $J_{2}^{j}$. Next let $\bar{\epsilon}_{0}>0$ be such that $A_{1} e^{-\sigma\left(\epsilon^{-1 / 4}-2\right)} \leq \epsilon$ for $0<\epsilon \leq \bar{\epsilon}_{0}$. Then, noting that $\gamma_{\xi}(t) \geq 0$, we get, for $s \in I_{1}^{j} \cup I_{3}^{j} \cup J_{1}^{j} \cup J_{3}^{j}$ and $0<\epsilon \leq \bar{\epsilon}_{0}$ :

$$
g\left(a \rho^{2}+\left(\gamma_{\xi}\left(t_{j}-s\right)+\rho\right)^{2}\right) \leq g\left(a \rho^{2}+(\epsilon+\rho)^{2}\right) .
$$

Hence:

$$
\int_{I_{1}^{j} \cup I_{3}^{j} \cup J_{1}^{j} \cup J_{3}^{j}} e^{-\sqrt{\epsilon} \delta s} g\left(a \rho^{2}+\left(\gamma_{\xi}\left(t_{j}-s\right)+\rho\right)^{2}\right) d s \leq \frac{g\left(a \rho^{2}+(\epsilon+\rho)^{2}\right)}{\sqrt{\epsilon} \delta}\left[1-e^{-2 m \sqrt{\epsilon} \delta}\right]
$$

Putting (66), (67) together we get

$$
\begin{array}{r}
\int_{0}^{2 m} e^{-\sqrt{\epsilon} \delta s} g\left(a \rho^{2}+\right. \\
\left.\left(\gamma_{\xi}\left(t_{j}-s\right)+\rho\right)^{2}\right) d s \leq 4 \frac{g\left(a \rho^{2}+(M+\rho)^{2}\right)}{\epsilon^{1 / 4}} \\
+\frac{g\left(a \rho^{2}+(\rho+\epsilon)^{2}\right)}{\sqrt{\epsilon} \delta}\left(1-e^{-2 m \sqrt{\epsilon} \delta}\right)
\end{array}
$$


To conclude the proof we simply have to observe that

$$
\begin{aligned}
& \int_{0}^{\infty} e^{-\sqrt{\epsilon} \delta s} g\left(a \rho^{2}+\left(\gamma_{\xi}(t-s)+\rho\right)^{2}\right) d s= \\
& \sum_{j=0}^{\infty} \int_{2 j m}^{2(j+1) m} e^{-\sqrt{\epsilon} \delta s} g\left(a \rho^{2}+\left(\gamma_{\xi}(t-s)+\rho\right)^{2}\right) d s= \\
& \sum_{j=0}^{\infty} e^{-2 \sqrt{\epsilon} \delta j m} \int_{0}^{2 m} e^{-\sqrt{\epsilon} \delta s} g\left(a \rho^{2}+\left(\gamma_{\xi}\left(t_{j}-s\right)+\rho\right)^{2}\right) d s
\end{aligned}
$$

and use (68). The proof is complete

Now we can state the following result.

4. Proposition. There exist $\nu_{0}, \rho_{0}, \epsilon_{0}$ and $m_{0}$ such that for any $\nu<\nu_{0}, \rho<\rho_{0}$, $\epsilon<\epsilon_{0}$ and $m>m_{0}$ we have

$$
\left\|T^{3}(u, \xi, m, \epsilon)\right\|_{Z} \leq \frac{8 i \nu}{\delta_{i}}+\frac{8 k}{\delta_{i}}\left[2 N \sqrt{\delta_{i}} \epsilon^{1 / 8}+N^{\prime}(3 \rho+\epsilon)\right]\|z\|_{Z} .
$$

Hence, in particular, we can assume that $T^{3}$ maps $U_{\rho}$ in $Z_{\rho}$.

Furthermore for any $\hat{u}, \bar{u} \in U_{\rho}$ and $\hat{\xi}=(E, \hat{\alpha}), \bar{\xi}=(E, \bar{\alpha})$, one has:

$$
\begin{aligned}
& \left\|T^{3}(\hat{u}, \hat{\xi}, m, \epsilon)-T^{3}(\bar{u}, \bar{\xi}, m, \epsilon)\right\|_{Z} \leq \frac{8 k N^{\prime}}{\delta_{i}} \times \\
& {\left[2\left(3 \rho+\epsilon+2 \sqrt{\delta_{i}} \epsilon^{1 / 8}(3 \rho+M)\right)\|\hat{u}-\bar{u}\|_{U}+M \rho \sigma\|\hat{\alpha}-\bar{\alpha}\|\right] .}
\end{aligned}
$$

Proof. Let $z_{j}(t)$ be the function defined in (64). First we observe that, for any $h_{j} \in L^{\infty}(\mathbb{R})$ we have, using Hölder inequality:

$$
\begin{aligned}
\frac{j^{2}}{4 i^{2}}\left|z_{j}(t)\right| & \leq \sqrt{\epsilon} \int_{0}^{\infty} e^{-\sqrt{\epsilon} \delta_{i} s / 2}\left|h_{j}(t-s)\right| d s \\
& \leq \sqrt{\epsilon}\left(\int_{0}^{\infty} e^{-\sqrt{\epsilon} \delta_{i} s / 2} d s\right)^{1 / 2}\left(\int_{0}^{\infty} e^{-\sqrt{\epsilon} \delta_{i} s / 2} h_{j}(t-s)^{2} d s\right)^{1 / 2} \\
& \leq\left(\frac{2 \sqrt{\epsilon}}{\delta_{i}}\right)^{1 / 2}\left(\int_{0}^{\infty} e^{-\sqrt{\epsilon} \delta_{i} s / 2} h_{j}(t-s)^{2} d s\right)^{1 / 2}
\end{aligned}
$$

Hence,

$$
j^{4}\left|z_{j}(t)\right|^{2} \leq \frac{32 i^{5} \sqrt{\epsilon}}{\delta} \int_{0}^{\infty} e^{-\sqrt{\epsilon} \delta_{i} s / 2} h_{j}(t-s)^{2} d s .
$$

Assuming that

$$
\|h\|^{2}:=\left\|\sum_{j>i} h_{j}^{2}(\cdot)\right\|_{\infty}<\infty
$$

from the Monotone Convergence Theorem we obtain:

$$
\left\|\left\{L_{j}^{z}\left(h_{j}\right)\right\}_{j>i}\right\|_{Z}^{2} \leq\left\|\sum_{j>i} j^{4} z_{j}^{2}\right\|_{\infty} \leq\left(\frac{8 i^{3}}{\delta}\right)^{2}\|h\|^{2} .
$$

Now, taking

$$
h_{j}(t)=\int_{0}^{\pi} h(x, t / i) \sqrt{\frac{2}{\pi}} \sin (j x) d x
$$


we have from Parseval equality:

$$
\sum_{j>i}\left|h_{j}(t)\right|^{2} \leq \int_{0}^{\pi} h(x, t)^{2} d x \leq 1
$$

Hence

$$
\left\|\left\{L_{j}^{z}\left[\nu_{i} \int_{0}^{\pi} h(x, t / i) \sqrt{\frac{2}{\pi}} \sin (j x) d x\right]\right\}_{j>i}\right\|_{Z} \leq 8 i^{2} \nu \delta^{-1} .
$$

Next we estimate $\left\|\left\{L_{j}^{z}\left[\mathfrak{F}(\xi, u, t) \frac{j^{2}}{i^{2}} z_{j}(t)\right]\right\}_{j>i}\right\|_{Z}$. To this end we observe that

$$
\mathfrak{F}(\xi, u, t) \frac{j^{2}}{i^{2}} z_{j}(t)=\frac{j}{i} F\left(\frac{j}{i} z_{j}(t), c_{j}(t)\right)
$$

where

$$
c_{j}(t)=\left[i^{-2} \sum_{l<i} l^{2} \phi_{l}^{2}(t)+\left(\gamma_{\xi}(t)+y(t)\right)^{2}+i^{-2} \sum_{\substack{p>i \\ p \neq j}} p^{2} z_{p}^{2}(t)\right]^{1 / 2} .
$$

Hence we have to estimate

$$
\sup _{t \in \mathbb{R}} \sum_{j>i} j^{4} \frac{4 \epsilon}{\omega_{j, \epsilon}^{2}}\left(\int_{0}^{\infty} e^{-\sqrt{\epsilon} \delta_{i} s / 2} \frac{j}{i} F\left(\frac{j}{i} z_{j}(t-s), c_{j}(t-s)\right) d s\right)^{2} .
$$

First we estimate the above quantity replacing $F$ with $|F|$. Then, from Hölder inequality we get

$$
\begin{aligned}
& \left(\int_{0}^{\infty} e^{-\sqrt{\epsilon} \delta_{i} s / 2}\left|F\left(\frac{j}{i} z_{j}(t-s), c_{j}(t-s)\right)\right| d s\right)^{2} \\
& \leq \int_{0}^{\infty} e^{-\sqrt{\epsilon} \delta_{i} s / 2} d s \cdot \int_{0}^{\infty} e^{-\sqrt{\epsilon} \delta_{i} s / 2} F\left(\frac{j}{i} z_{j}(t-s), c_{j}(t-s)\right)^{2} d s \\
& =\frac{2}{\delta_{i} \sqrt{\epsilon}} \int_{0}^{\infty} e^{-\sqrt{\epsilon} \delta_{i} s / 2} F\left(\frac{j}{i} z_{j}(t-s), c_{j}(t-s)\right)^{2} d s
\end{aligned}
$$

Thus plugging this into (71) and using $\omega_{j, \epsilon} \geq \frac{j^{2}}{2 i^{2}}$ we see that we need to estimate:

$$
\frac{32 i \sqrt{\epsilon}}{\delta} \sup _{t \in \mathbb{R}} \sum_{j>i} i^{2} j^{2} \int_{0}^{\infty} e^{-\sqrt{\epsilon} \delta_{i} s / 2} F\left(\frac{j}{i} z_{j}(t-s), c_{j}(t-s)\right)^{2} d s .
$$

Now, setting $f_{\max }(x)=\sup \{f(t) \mid 0 \leq t \leq x\}$, and noting that $c_{j}^{2}(t)+$ $\frac{j^{2}}{i^{2}} z_{j}(t)^{2} \leq 2 \rho^{2}+\left(\gamma_{\xi}(t)+\rho\right)^{2}$, we see that

$$
\begin{aligned}
& \sum_{j>i} i^{2} j^{2} \int_{0}^{\infty} e^{-\sqrt{\epsilon} \delta_{i} s / 2} F\left(\frac{j}{i} z_{j}(t-s), c_{j}(t-s)\right)^{2} d s \leq \\
& \sum_{j>i} i^{2} j^{2} \int_{0}^{\infty} e^{-\sqrt{\epsilon} \delta_{i} s / 2} f_{\max }^{2}\left(2 \rho^{2}+\left(\gamma_{\xi}(t-s)+\rho\right)^{2}\right) \frac{j^{2}}{i^{2}} z_{j}^{2}(t-s) d s \\
& \leq \int_{0}^{\infty} e^{-\sqrt{\epsilon} \delta_{i} s / 2} f_{\max }^{2}\left(2 \rho^{2}+\left(\gamma_{\xi}(t-s)+\rho\right)^{2}\right) d s\|z\|_{Z}^{2}
\end{aligned}
$$


having used the Monotone Convergence Theorem. But since $f_{\max }$ is positive and increasing, so is $f_{\max }^{2}$ and hence Lemma 6 gives:

$$
\begin{aligned}
& \int_{0}^{\infty} e^{-\sqrt{\epsilon} \delta_{i} s / 2} f_{\max }^{2}\left(2 \rho^{2}+\left(\gamma_{\xi}(t-s)+\rho\right)^{2}\right) d s \\
& \leq 4 \frac{f_{\max }^{2}\left(2 \rho^{2}+(M+\rho)^{2}\right)}{\epsilon^{1 / 4}\left(1-e^{-m \delta_{i} \sqrt{\epsilon}}\right)}+2 \frac{f_{\max }^{2}\left(2 \rho^{2}+(\epsilon+\rho)^{2}\right)}{\sqrt{\epsilon} \delta_{i}} \\
& \leq 4 \frac{N^{2}}{\epsilon^{1 / 4}\left(1-e^{-m \delta_{i} \sqrt{\epsilon}}\right)}+2 \frac{N^{\prime 2}\left[2 \rho^{2}+(\epsilon+\rho)^{2}\right]}{\sqrt{\epsilon} \delta_{i}}
\end{aligned}
$$

since from $0 \leq f\left(x^{2}\right) \leq N^{\prime}|x|$ (see (15)) we get $f_{\max }^{2}\left(x^{2}\right) \leq N^{\prime 2} x^{2}$. Then (69) follows from $e^{-m \delta_{i} \sqrt{\epsilon}}<e^{-\delta_{i} \epsilon^{-1 / 4}} \leq \frac{1}{2}$ (provided we take $0<\epsilon \leq\left(\frac{\delta_{i}}{\ln 2}\right)^{4}$ ) and the inequality $\sqrt{a^{2}+b^{2}} \leq|a|+|b|$.

Now we prove (70). To this end we need to estimate

$$
\left\{L_{j}^{z}\left[\mathfrak{F}(\hat{\xi}, \hat{u}, t) \frac{j^{2}}{i^{2}} \hat{z}_{j}(t)-\mathfrak{F}(\bar{\xi}, \bar{u}, t) \frac{j^{2}}{i^{2}} \bar{z}_{j}(t)\right]\right\}_{j>i} .
$$

We have

$$
\begin{aligned}
\mathfrak{F}(\hat{\xi}, \hat{u}, t) \frac{j^{2}}{i^{2}} \hat{z}_{j}(t)-\mathfrak{F}(\bar{\xi}, \bar{u}, t) \frac{j^{2}}{i^{2}} \bar{z}_{j}(t) & =\frac{j}{i}\left[F\left(\frac{j}{i} \hat{z}_{j}(t), \hat{c}_{j}(t)\right)-F\left(\frac{j}{i} \hat{z}_{j}(t), \bar{c}_{j}(t)\right)\right] \\
& +\frac{j}{i}\left[F\left(\frac{j}{i} \hat{z}_{j}(t), \bar{c}_{j}(t)\right)-F\left(\frac{j}{i} \bar{z}_{j}(t), \bar{c}_{j}(t)\right)\right] .
\end{aligned}
$$

Next, using again Hölder inequality, $\left|F_{\zeta}(\eta, \zeta)\right| \leq N^{\prime}|\eta|$ and the Monotone Convergence Theorem:

$$
\begin{aligned}
& \sum_{j>i} j^{4}\left[L_{j}^{z}\left\{\frac{j}{i}\left[F\left(\frac{j}{i} \hat{z}_{j}, \hat{c}_{j}(t)\right)-F\left(\frac{j}{i} \hat{z}_{j}, \bar{c}_{j}(t)\right)\right]\right\}\right]^{2} \leq \\
& \leq \sum_{j>i} \frac{32 \sqrt{\epsilon} i^{2} j^{2}}{\delta_{i}} \int_{0}^{\infty} e^{-\sqrt{\epsilon} \delta_{i} s / 2}\left[N^{\prime} \frac{j}{i}\left|\hat{z}_{j}(t-s)\right| \mid \hat{c}_{j}(t-s)-\bar{c}_{j}(t-s)\right]^{2} d s \\
& \leq \frac{32 N^{\prime 2} \sqrt{\epsilon}}{\delta_{i}} \int_{0}^{\infty} e^{-\sqrt{\epsilon} \delta_{i} s / 2} \sum_{j>i} j^{4}\left|\hat{z}_{j}(t-s)\right|^{2} d s\left[\|\hat{u}-\bar{u}\|_{U}+\left\|\gamma_{\hat{\xi}}-\gamma_{\bar{\xi}}\right\|_{\infty}\right]^{2} \\
& \leq \frac{64 N^{\prime 2}}{\delta_{i}^{2}}\|\hat{z}\|^{2}\left[\|\hat{u}-\bar{u}\|_{U}+\left\|\gamma_{\hat{\xi}}-\gamma_{\bar{\xi}}\right\|_{\infty}\right]^{2} .
\end{aligned}
$$

As a consequence

$$
\begin{aligned}
& \left\|\left\{L_{j}^{z}\left\{\frac{j}{i}\left[F\left(\frac{j}{i} \hat{z}_{j}, \hat{c}_{j}(t)\right)-F\left(\frac{j}{i} \hat{z}_{j}, \bar{c}_{j}(t)\right)\right]\right\}\right\}_{j>i}\right\|_{Z} \\
& \leq \frac{8 N^{\prime}}{\delta_{i}} \rho\left[\|\hat{u}-\bar{u}\|_{U}+\left\|\gamma_{\hat{\xi}}-\gamma_{\bar{\xi}}\right\|_{\infty}\right] .
\end{aligned}
$$

Next, we look at

$$
\sum_{j>i} j^{4}\left[L_{j}^{z}\left\{\frac{j}{i}\left(F\left(\frac{j}{i} \hat{z}_{j}, \bar{c}_{j}(t)\right)-F\left(\frac{j}{i} \bar{z}_{j}, \bar{c}_{j}(t)\right)\right)\right\}\right]^{2}
$$


First we observe that from Lemma 1 and $j^{2}\left\|z_{j}\right\|_{\infty} \leq\|z\| \leq \rho$, it follows:

$$
\begin{aligned}
& \left|F\left(\frac{j}{i} \hat{z}_{j}(t), \bar{c}_{j}(t)\right)-F\left(\frac{j}{i} \bar{z}_{j}(t), \bar{c}_{j}(t)\right)\right| \\
& \leq \int_{0}^{1} \mid F_{\eta}\left(\frac{j}{i}\left[\theta \hat{z}_{j}(t)+(1-\theta) \bar{z}_{j}(t), \bar{c}_{j}(t)\right)\left|d \theta \frac{j}{i}\right| \hat{z}_{j}(t)-\bar{z}_{j}(t) \mid\right. \\
& \leq N^{\prime} \int_{0}^{1}\left[\frac{1}{i j} \rho+\sqrt{\frac{1}{i^{2} j^{2}}} \rho^{2}+\bar{c}_{j}^{2}(t)\right] d \theta \frac{j}{i}\left|\hat{z}_{j}(t)-\bar{z}_{j}(t)\right| \\
& \leq N^{\prime}\left[\rho+\sqrt{\left.\rho^{2}+\bar{c}_{j}^{2}(t)\right]} \frac{j}{i}\left|\hat{z}_{j}(t)-\bar{z}_{j}(t)\right|\right. \\
& \leq N^{\prime}\left[\rho+\sqrt{\left.5 \rho^{2}+2 \gamma_{\xi}^{2}(t)\right]} \frac{j}{i}\left|\hat{z}_{j}(t)-\bar{z}_{j}(t)\right|\right.
\end{aligned}
$$

since

$$
c_{j}^{2}(t) \leq \sum_{l<i} \phi_{l}(t)^{2}+2 y(t)^{2}+2 \gamma_{\xi}^{2}(t)+\sum_{j>i} j^{4} z_{j}^{2}(t) \leq 4 \rho^{2}+2 \gamma_{\xi}^{2}(t) .
$$

Then, again from Hölder inequality and the Monotone Convergence Theorem:

$$
\begin{aligned}
& \sum_{j>i} j^{4}\left[L_{j}^{z}\left\{\frac{j}{i}\left(F\left(\frac{j}{i} \hat{z}_{j}(t), \bar{c}_{j}(t)\right)-F\left(\frac{j}{i} \bar{z}_{j}(t), \bar{c}_{j}(t)\right)\right)\right\}\right]^{2} \\
& \leq \frac{32 \sqrt{\epsilon}}{\delta_{i}} \sum_{j>i} i^{2} j^{2} \times \\
& \int_{0}^{\infty} e^{-\sqrt{\epsilon} \delta_{i} s / 2}\left[F\left(\frac{j}{i} \hat{z}_{j}(t-s), \bar{c}_{j}(t-s)\right)-F\left(\frac{j}{i} \bar{z}_{j}(t-s), \bar{c}_{j}(t-s)\right)\right]^{2} d s \\
& \leq \frac{32 N^{\prime 2} \sqrt{\epsilon}}{\delta_{i}} \sum_{j>i} \int_{0}^{\infty} e^{-\sqrt{\epsilon} \delta_{i} s / 2} \times \\
& \leq \frac{32 N^{\prime 2} \sqrt{\epsilon}}{\delta_{i}} \int_{0}^{\infty} e^{-\sqrt{\epsilon} \delta_{i} s / 2}\left[\rho+\sqrt{5 \rho^{2}+2 \gamma_{\bar{\xi}}^{2}(t-s)}\right]^{2} d s\|\hat{z}-\bar{z}\|_{Z}^{2} \\
& \leq \frac{128 N^{\prime 2} \sqrt{\epsilon}}{\delta_{i}} \int_{0}^{\infty} e^{-\sqrt{\epsilon} \delta_{i} s / 2}\left[2 \rho^{2}+\left(\gamma_{\bar{\xi}}(t-s)+\rho\right)^{2}\right] d s\|\hat{z}-\bar{z}\|_{Z}^{2}
\end{aligned}
$$

since $\left[\rho+\sqrt{5 \rho^{2}+2 \gamma_{\bar{\xi}}^{2}(t)}\right]^{2} \leq 4\left(3 \rho^{2}+\gamma_{\bar{\xi}}^{2}(t)\right)<4\left(2 \rho^{2}+\left(\gamma_{\bar{\xi}}(t)+\rho\right)^{2}\right)$. Then from Lemma 6 we obtain

$$
\begin{aligned}
& \left\|\left\{L_{j}^{z}\left\{\frac{j}{i}\left[F\left(\frac{j}{i} \hat{z}_{j}(t), \bar{c}_{j}(t)\right)-F\left(\frac{j}{i} \bar{z}_{j}(t), \bar{c}_{j}(t)\right)\right]\right\}\right\}_{j>i}\right\|_{Z} \\
& \left.\leq \frac{16 N^{\prime}}{\delta_{i}}\left[4 \delta_{i}\left(2 \rho^{2}+(M+\rho)^{2}\right) \epsilon^{1 / 4}+2 \rho^{2}+(\rho+\epsilon)^{2}\right)\right]^{1 / 2}\|\hat{z}-\bar{z}\|_{Z}
\end{aligned}
$$

Putting (72) and (73) together and recalling (17) and the fact that $\sqrt{a^{2}+b^{2}} \leq$ $|a|+|b|$ we obtain (70). The proof is complete. 
Now putting together Propositions 2, 3 and 4 we find that, if $\rho, \epsilon, \nu$ and $\frac{1}{m}$ are small enough, the operator

$$
T(u, \xi, m, \epsilon)=\left(T^{1}(u, \xi, m, \epsilon), T^{2}(u, \xi, m, \epsilon), T^{3}(u, \xi, m, \epsilon)\right)
$$

maps $U_{\rho} \times X \times \mathbb{N}_{m_{0}} \times \mathbb{R}_{+}$into $U_{\rho}$. Furthermore $T$ is a contraction in $U_{\rho}$ and the contraction factor tends to 0 as $\epsilon+\nu+\rho \rightarrow 0$.

So we can apply Banach Fixed Point Theorem to get the following

2. Theorem. Assume that the conditions (F1)-(F2) hold and fix $\delta>0$. Then there exist positive numbers $\rho_{0}>0, \epsilon_{0}>0$, and $\nu_{0}>0$ such that for any $\xi \in X$, $0<\epsilon<\epsilon_{0},|\nu|<\nu_{0}, m>\epsilon^{-3 / 4}$, the integro-differential system (30)-(32) has a unique bounded solution

$$
u(\xi, \nu, \epsilon, m ; t)=(\phi(\xi, \nu, \epsilon, m ; t), y(\xi, \nu, \epsilon, m ; t), z(\xi, \nu, \epsilon, m ; t)) \in U_{\rho_{0}} .
$$

Moreover

$$
\|u(\xi, \nu, \epsilon, m ; \cdot)\|_{U} \leq C(|\nu|+\epsilon)
$$

for a suitable constant $C$ independent of $(\nu, \epsilon, \xi)$. Finally, $\phi(\xi, \nu, \epsilon, m ; \cdot), y(\xi, \nu, \epsilon, m ; \cdot)$, $z(\xi, \nu, \epsilon, m ; \cdot)$ are Lipschitz-continuous in $\alpha$ uniformly with respect to $(E, m)$. More precisely there exists a positive constant $\bar{c}$ such that

$$
\|u(\hat{\xi}, \nu, \epsilon, m ; \cdot)-u(\bar{\xi}, \nu, \epsilon, m ; \cdot)\|_{U} \leq \bar{c}(|\nu|+\epsilon)\|\hat{\alpha}-\bar{\alpha}\| .
$$

Proof. As we have already observed, from Propositions 2, 3 and 4 it follows immediately that $\rho_{0}>0, \epsilon_{0}>0$ and $\nu_{0}>0$ exist such that $T=\left(T^{1}, T^{2}, T^{3}\right)$ : $U_{\rho} \times X \times \mathbb{N}_{m_{0}} \times \mathbb{R}_{+} \rightarrow U_{\rho}$ (with $m_{0}>\epsilon^{-3 / 4}$ ) is a contraction in $u \in U_{\rho}$ uniform with respect to $(\xi, m, \epsilon)$. Moreover if $\rho_{0}>0, \epsilon_{0}>0$ and $\nu_{0}>0$ are sufficiently small, from Propositions 2, 3 and 4 we see that we can write:

$$
\begin{aligned}
\|y(\xi, \ldots)\|_{Y} & \leq \frac{1}{4}\|u(\xi, \ldots)\|_{U}+k_{4}(|\nu|+\epsilon) \\
\|\phi(\xi, \ldots)\|_{\Phi} & \leq \frac{1}{4}\|\phi(\xi, \ldots)\|_{\Phi}+2|\nu| \sqrt{\epsilon} \\
\|z(\xi, \ldots)\|_{Y} & \leq \frac{1}{4}\|z(\xi, \ldots)\|_{Z}+8 i^{2}|\nu| \delta^{-1} .
\end{aligned}
$$

Thus we get, immediately:

$$
\|\phi(\xi, \ldots)\|_{\Phi} \leq \frac{8}{3}|\nu| \sqrt{\epsilon} \quad \text { and } \quad\|z(\xi, \ldots)\|_{Z} \leq \frac{32}{3} i^{2}|\nu| \delta^{-1} .
$$

Then:

$$
\begin{aligned}
\frac{3}{4}\|y(\xi, \ldots)\|_{Y} & \leq \frac{1}{4}\left[\|\phi(\xi, \ldots)\|_{\Phi}+\|z(\xi, \ldots)\|_{Z}\right]+k_{4}(|\nu|+\epsilon) \\
& \leq \frac{2}{3}|\nu| \sqrt{\epsilon}+\frac{8}{3} i^{2}|\nu| \delta^{-1}+k_{4}(|\nu|+\epsilon)
\end{aligned}
$$

that is

$$
\|y(\xi, \ldots)\|_{Y} \leq \frac{8}{9}|\nu| \sqrt{\epsilon}+\frac{32}{9} i^{2}|\nu| \delta^{-1}+\frac{4}{3} k_{4}(|\nu|+\epsilon) .
$$


Plugging everything together we obtain (74). Then, again from Propositions 2, 3 and 4 , we get

$$
\begin{aligned}
\|y(\hat{\xi}, \ldots)-y(\hat{\xi}, \ldots)\|_{Y} & \leq \frac{1}{4}\|u(\hat{\xi}, \ldots)-u(\bar{\xi}, \ldots)\|_{U} \\
& +[O(|\nu|+\epsilon+\rho \sqrt{\epsilon})+\rho \Delta(\rho)]\|\hat{\alpha}-\bar{\alpha}\| \\
\|\phi(\hat{\xi}, \ldots)-\phi(\bar{\xi}, \ldots)\|_{\Phi} & \leq \frac{1}{4}\|u(\hat{\xi}, \ldots)-u(\bar{\xi}, \ldots)\|_{U}+\widetilde{c}_{1} \rho \sqrt{\epsilon}\|\hat{\alpha}-\bar{\alpha}\| \\
\|z(\hat{\xi}, \ldots)-z(\bar{\xi}, \ldots)\|_{Z} & \leq \frac{1}{4}\|u(\hat{\xi}, \ldots)-u(\bar{\xi}, \ldots)\|_{U}+\widetilde{c}_{2} \rho\|\hat{\alpha}-\bar{\alpha}\|
\end{aligned}
$$

where $\rho=\max \left\{\|u(\hat{\xi}, \ldots)\|_{U},\|u(\bar{\xi}, \ldots)\|_{U}\right\} \leq C(|\nu|+\epsilon)$. Then (75) easily follows.

Let us denote by

$$
\begin{aligned}
& G(\xi, m, \nu, \epsilon):=\left(\mathbf{I}-Q_{m, \xi}\right)\left\{\sqrt{\epsilon} \delta_{i} \dot{\gamma}_{\xi}(t)\right. \\
& \left.-\frac{2}{\sqrt{\pi}} \nu_{i} \int_{0}^{\pi} h(x, t / i) \sin (i x) d x+r(\xi, \epsilon, \nu, \delta, m ; t)\right\}
\end{aligned}
$$

where

$$
\begin{aligned}
& r(\xi, \epsilon, \nu, \delta, m ; t):=-k\left[2 f^{\prime}\left(\gamma_{\xi}(t)^{2}\right) \gamma_{\xi}(t)^{2}+f\left(\gamma_{\xi}(t)^{2}\right)\right] y(t)+ \\
& +k \mathfrak{F}(\xi, u, t)\left[\gamma_{\xi}(t)+y(t)\right]-k f\left(\gamma_{\xi}(t)^{2}\right) \gamma_{\xi}(t)-\sqrt{\epsilon} \delta_{i} \dot{y}(t) \\
& =k h_{2}(\xi, u(\xi, \nu, \epsilon, m ; t) ; t)-\sqrt{\epsilon} \delta_{i} \dot{y}(t) .
\end{aligned}
$$

$h_{2}(\xi, u ; t)$ being the function defined in (34). Now following [1] we see that in order to find a bounded solution near $\gamma_{\xi}$ of the original equations (8)-(10) we need to show that the equation $G(\xi, m, \nu, \epsilon)=0$ can be solved for some values of the parameters. From (41) we find then

$$
\|r(\xi, \epsilon, \nu, \delta, m ; \cdot)\|_{\infty} \leq \frac{k}{2} \max \left\{k_{2}, k_{3}, N^{\prime}\right\}\|u(\xi, \nu, \epsilon, m ; \cdot)\|_{U}^{2}+\sqrt{\epsilon} \delta_{i}\|y(\xi, \nu, \epsilon, m ; \cdot)\|_{Y}
$$

Setting $\nu=\sqrt{\epsilon} \mu$ we see, according to (74), that

$$
\|r(\xi, \epsilon, \sqrt{\epsilon} \mu, \delta, m ; \cdot)\|_{\infty}=O(\epsilon)
$$

as $\epsilon \rightarrow 0$ uniformly with respect to $(\xi, m, \mu, \delta)$ provided $(\mu, \delta)$ belongs to a fixed compact subset of $\mathbb{R}^{2}$ and $m \geq m_{0}$.

Next, let $\hat{\xi}=(E, \hat{\alpha}), \bar{\xi}=(E, \bar{\alpha}) \in X$. Then:

$$
\begin{aligned}
& |r(\hat{\xi}, \epsilon, \nu, \delta, m ; t)-r(\bar{\xi}, \epsilon, \nu, \delta, m ; t)| \\
& \leq k\left|h_{2}(\hat{\xi}, u(\hat{\xi}, \nu, \epsilon, m ; t) ; t)-h_{2}(\hat{\xi}, u(\bar{\xi}, \nu, \epsilon, m ; t) ; t)\right| \\
& +k\left|h_{2}(\hat{\xi}, u(\bar{\xi}, \nu, \epsilon, m ; t) ; t)-h_{2}(\bar{\xi}, u(\bar{\xi}, \nu, \epsilon, m ; t) ; t)\right| \\
& +\sqrt{\epsilon} \delta_{i}|\dot{y}(\hat{\xi}, \nu, \epsilon, m ; t)-\dot{y}(\bar{\xi}, \nu, \epsilon, m ; t)| .
\end{aligned}
$$

Now, from (43), (44) with $\rho=\max \left\{\|u(\hat{\xi}, \nu, \epsilon, m ; \cdot)\|_{U},\|u(\bar{\xi}, \nu, \epsilon, m ; \cdot)\|_{U}\right\}$ we get:

$$
\begin{aligned}
& \left|h_{2}(\hat{\xi}, u(\hat{\xi}, \nu, \epsilon, m ; t) ; t)-h_{2}(\hat{\xi}, u(\bar{\xi}, \nu, \epsilon, m ; t) ; t)\right| \leq 3 \max \left\{k_{2}, k_{3}, N^{\prime}\right\} \times \\
& \max \left\{\|u(\hat{\xi}, \nu, \epsilon, m ; \cdot)\|_{U},\|u(\bar{\xi}, \nu, \epsilon, m ; \cdot)\|_{U}\right\}\|u(\hat{\xi}, \nu, \epsilon, m ; \cdot)-u(\bar{\xi}, \nu, \epsilon, m ; \cdot)\|_{U}
\end{aligned}
$$

and, from (51) with $\rho=\|u(\bar{\xi}, \nu, \epsilon, m ; \cdot)\|_{U}$ :

$$
\begin{aligned}
& \left|h_{2}(\hat{\xi}, u(\bar{\xi}, \nu, \epsilon, m ; t) ; t)-h_{2}(\bar{\xi}, u(\bar{\xi}, \nu, \epsilon, m ; t) ; t)\right| \leq 2\|u(\bar{\xi}, \nu, \epsilon, m ; \cdot)\|_{U} \times \\
& \widetilde{\Delta}\left(\|u(\bar{\xi}, \nu, \epsilon, m ; \cdot)\|_{U}\right)\|\hat{\alpha}-\bar{\alpha}\| .
\end{aligned}
$$


Thus from (74), (75) we finally obtain:

$$
\|r(\hat{\xi}, \epsilon, \sqrt{\epsilon} \mu, \delta, m ; \cdot)-r(\bar{\xi}, \epsilon, \sqrt{\epsilon} \mu, \delta, m ; \cdot,)\|_{\infty} \leq \sqrt{\epsilon} O(1)\|\hat{\alpha}-\bar{\alpha}\|
$$

where $o(1) \rightarrow 0$ as $\epsilon \rightarrow 0$, uniformly with respect to $(\mu, \delta, E, m)$ provided $(\mu, \delta)$ belongs to a fixed compact subset of $\mathbb{R}^{2}, m>m_{0}$.

Next, as it has been proved in [1, Proposition 2], the equation $\frac{G(\xi, m, \sqrt{\epsilon} \mu, \epsilon)}{\sqrt{\epsilon}}=0$ is equivalent to $\widetilde{G}(\xi, m, \mu, \epsilon)=0$, where

$$
\begin{aligned}
& \widetilde{G}(\xi, m, \mu, \epsilon):= \\
& {\left[\mathcal{L}_{m, \xi}+N_{m, \xi}\right]\left\{\delta \dot{\gamma}_{\xi}(t)-\mu_{i} \sqrt{\frac{2}{\pi}} \int_{0}^{\pi} h(x, t / i) \sin (i x) d x+\frac{1}{\sqrt{\epsilon}} r(\xi, \epsilon, \sqrt{\epsilon} \mu, \delta, m ; t)\right\}}
\end{aligned}
$$

where $\mu_{i}=\mu / i$ and $\mathcal{L}_{m, \xi}: L^{\infty}(\mathbb{R}) \rightarrow \ell_{E}^{\infty}$ is a linear operator such that

$$
\begin{aligned}
& \left\|\mathcal{L}_{m, \xi}\right\| \leq A e^{-m \sigma} \\
& \left\|\mathcal{L}_{m, \xi^{\prime \prime}}-\mathcal{L}_{m, \xi^{\prime}}\right\| \leq A e^{-m \sigma}\left\|\alpha^{\prime \prime}-\alpha^{\prime}\right\|
\end{aligned}
$$

for a suitable positive constant $A$ and $N_{m, \xi}: L^{\infty}(\mathbb{R}) \rightarrow \ell_{E}^{\infty}$ is a nonlinear operator that satisfies

$$
\begin{aligned}
& \left\|N_{m, \xi}\right\| \leq c_{N} \\
& \left\|N_{m, \xi^{\prime \prime}}-N_{m, \xi^{\prime}}\right\| \leq c_{N}\left\|\alpha^{\prime \prime}-\alpha^{\prime}\right\|
\end{aligned}
$$

for some positive constant $c_{N}$. Then, from (76), (77), (78), (79) we see that, for $m>\epsilon^{-3 / 4}$,

$$
\widetilde{G}(\xi, m, \mu, \epsilon)=N_{m, \xi}\left\{\delta_{i} \dot{\gamma}_{\xi}(t)-\mu_{i} \sqrt{\frac{2}{\pi}} \int_{0}^{\pi} h(x, t / i) \sin (i x) d x\right\}+O(\sqrt{\epsilon})
$$

and, using also (17),

$$
\begin{aligned}
& \widetilde{G}\left(\xi^{\prime \prime}, m, \mu, \epsilon\right)-\widetilde{G}\left(\xi^{\prime}, m, \mu, \epsilon\right)=N_{m, \xi^{\prime \prime}}\left\{\delta_{i} \dot{\gamma}_{\xi^{\prime \prime}}(t)-\mu_{i} \sqrt{\frac{2}{\pi}} \int_{0}^{\pi} h(x, t / i) \sin (i x) d x\right\} \\
& -N_{m, \xi^{\prime}}\left\{\delta_{i} \dot{\gamma}_{\xi^{\prime}}(t)-\mu_{i} \sqrt{\frac{2}{\pi}} \int_{0}^{\pi} h(x, t / i) \sin (i x) d x\right\}+o(1)\left\|\alpha^{\prime \prime}-\alpha^{\prime}\right\|
\end{aligned}
$$

as $\epsilon \rightarrow 0$, where $o(1)$ is uniform with respect to $(\xi, m, \mu)$. Now, let $\alpha=\left\{\alpha_{j}\right\}_{j \in \mathbb{Z}}, \alpha^{\prime}=$ $\left\{\alpha_{j}^{\prime}\right\}_{j \in \mathbb{Z}}, \alpha^{\prime \prime}=\left\{\alpha_{j}^{\prime \prime}\right\}_{j \in \mathbb{Z}} \in \ell_{E}^{\infty}$ and set $\xi=(E, \alpha), \xi^{\prime}=\left(E, \alpha^{\prime}\right), \xi^{\prime \prime}=\left(E, \alpha^{\prime \prime}\right)$. We define the following Melnikov-like function of $\tau \in \mathbb{R}$ :

$$
M_{\mu}(\tau)=\delta_{i} \int_{-\infty}^{\infty} \dot{\gamma}(t)^{2} d t-\mu_{i} \sqrt{\frac{2}{\pi}} \int_{-\infty}^{\infty} \int_{0}^{\pi} \dot{\gamma}(t)^{2} h(x,(t+\tau) / i) \sin (i x) d x d t
$$

and set

$$
\widetilde{\mathcal{M}}(\alpha)=\left\{e_{j} M_{\mu}\left(\alpha_{j}\right)\right\}_{j \in \mathbb{Z}}
$$

Now we prove that the function of $\alpha \in \ell_{E}^{\infty}$

$$
D(E, \alpha, m, \mu, \delta, \epsilon):=N_{m, \xi}\left\{\delta_{i} \dot{\gamma}_{\xi}(t)-\mu_{i} \sqrt{\frac{2}{\pi}} \int_{0}^{\pi} h(x, t / i) \sin (i x) d x\right\}-\widetilde{\mathcal{M}}(\alpha)
$$

satisfies

$$
D(E, \alpha, m, \mu, \delta, \epsilon)=O(\epsilon)
$$


and

$$
\|D(E, \hat{\alpha}, m, \mu, \delta, \epsilon)-D(E, \bar{\alpha}, m, \mu, \delta, \epsilon)\|=O(\epsilon)\|\hat{\alpha}-\bar{\alpha}\|
$$

as $\epsilon \rightarrow 0$ uniformly with respect to $(E, m, \mu, \delta)$ provided $(\mu, \delta)$ belongs to a fixed compact subset of $\mathbb{R}^{2}$.

In fact it is easily seen (see $[\mathbf{1}$, p. 195]) that the $j$ th component of the above map consists of the sum of the following two terms:

$$
\begin{aligned}
& -e_{j} \int_{-\infty}^{-m-\alpha_{j}} \dot{\gamma}(t)\left[\delta_{i} \dot{\gamma}(t)-\mu_{i} \sqrt{\frac{2}{\pi}} \int_{0}^{\pi} h\left(x,\left(t+\alpha_{j}\right) / i\right) \sin (i x) d x\right] d t \\
& =-e_{j} \int_{-\infty}^{-m} \dot{\gamma}\left(t-\alpha_{j}\right)\left[\delta_{i} \dot{\gamma}\left(t-\alpha_{j}\right)-\mu_{i} \sqrt{\frac{2}{\pi}} \int_{0}^{\pi} h(x, t / i) \sin (i x) d x\right] d t
\end{aligned}
$$

and

$$
\begin{aligned}
& -e_{j} \int_{m+\alpha_{j}}^{\infty} \dot{\gamma}(t)\left[\delta_{i} \dot{\gamma}(t)-\mu_{i} \sqrt{\frac{2}{\pi}} \int_{0}^{\pi} h\left(x,\left(t+\alpha_{j}\right) / i\right) \sin (i x) d x\right] d t \\
& =-e_{j} \int_{m}^{\infty} \dot{\gamma}\left(t+\alpha_{j}\right)\left[\delta_{i} \dot{\gamma}\left(t+\alpha_{j}\right)-\mu_{i} \sqrt{\frac{2}{\pi}} \int_{0}^{\pi} h(x, t / i) \sin (i x) d x\right] d t
\end{aligned}
$$

Now, from Hölder inequality, $\left|\alpha_{j}\right| \leq 2$ and $|\dot{\gamma}(t)| \leq \sigma \gamma(t) \leq \gamma(t) \leq A_{1} e^{-\sigma|t|}$, we obtain:

and similarly:

$$
\left|\int_{m}^{\infty} \dot{\gamma}\left(t+\alpha_{j}\right) d t\right| \leq \int_{m}^{\infty} \gamma\left(t+\alpha_{j}\right) d t \leq \frac{A_{1}}{\sigma} e^{2 \sigma} e^{-\sigma m}
$$

$$
\left|\int_{m}^{\infty} \dot{\gamma}^{2}\left(t+\alpha_{j}\right) d t\right| \leq \int_{m}^{\infty} A_{1}^{2} e^{-2 \sigma\left(t+\alpha_{j}\right)} d t \leq \frac{A_{1}^{2}}{2 \sigma} e^{4 \sigma} e^{-2 \sigma m}
$$

Since similar estimates hold for the other two terms we see that (80) follows since $m \geq \epsilon^{-3 / 4}$. As for the Lipschitz continuity of $D(E, \alpha, m, \mu, \delta, \epsilon)$ we observe that from the fact that $\gamma(t)$ satisfies equation (16) it follows $|\ddot{\gamma}(t)| \leq\left(k N+\sigma^{2}\right) \gamma(t)$ and hence

$$
\begin{aligned}
& \left|\int_{m}^{\infty}\left[\dot{\gamma}\left(t+\hat{\alpha}_{j}\right)-\dot{\gamma}\left(t+\bar{\alpha}_{j}\right)\right] d t\right| \\
& \leq \int_{m}^{\infty}\left|\int_{t+\bar{\alpha}_{j}}^{t+\hat{\alpha}_{j}}\right| \ddot{\gamma}(\tau)|d \tau| d t \leq\left(k N+\sigma^{2}\right) \frac{A_{1}}{\sigma^{2}} e^{2 \sigma} e^{-\sigma m}\left|\hat{\alpha}_{j}-\bar{\alpha}_{j}\right|
\end{aligned}
$$

since:

$$
\begin{aligned}
\int_{m}^{\infty}\left|\int_{t+\bar{\alpha}_{j}}^{t+\hat{\alpha}_{j}} \gamma(\tau) d \tau\right| d t & \leq \frac{A_{1}}{\sigma} \int_{m}^{\infty}\left|e^{-\sigma\left(t+\hat{\alpha}_{j}\right)}-e^{-\sigma\left(t+\bar{\alpha}_{j}\right)}\right| d t \\
& \leq \frac{A_{1}}{\sigma^{2}} e^{-\sigma m}\left|e^{-\sigma \hat{\alpha}_{j}}-e^{-\sigma \bar{\alpha}_{j}}\right|
\end{aligned}
$$

having used $t \geq m \geq 3>\left|\hat{\alpha}_{j}\right|,\left|\bar{\alpha}_{j}\right|$. Similarly,

$$
\begin{aligned}
& \left|\int_{m}^{\infty} \dot{\gamma}^{2}\left(t+\hat{\alpha}_{j}\right)-\dot{\gamma}^{2}\left(t+\bar{\alpha}_{j}\right) d t\right| \leq 2 M \int_{m}^{\infty}\left|\int_{t+\bar{\alpha}_{j}}^{t+\hat{\alpha}_{j}}\right| \ddot{\gamma}(\tau)|d \tau| d t \\
& \leq 2 M\left(k N+\sigma^{2}\right) \frac{A_{1}}{\sigma^{2}} e^{2 \sigma} e^{-\sigma m}\left|\hat{\alpha}_{j}-\bar{\alpha}_{j}\right|
\end{aligned}
$$


Since similar estimates hold for the other two terms (81) follows.

Thus:

$$
\widetilde{G}(\xi, m, \mu, \epsilon)=\widetilde{\mathcal{M}}(\alpha)+o(1)
$$

and

$$
\widetilde{G}\left(\xi^{\prime \prime}, m, \mu, \epsilon\right)-\widetilde{G}\left(\xi^{\prime}, m, \mu, \epsilon\right)=\left[\widetilde{\mathcal{M}}\left(\alpha^{\prime \prime}\right)-\widetilde{\mathcal{M}}\left(\alpha^{\prime}\right)\right]+o(1)\left\|\alpha^{\prime \prime}-\alpha^{\prime}\right\|
$$

Finally, again from $[\mathbf{1}]$ we know that $\widetilde{\mathcal{M}}(\alpha)$ is $C^{1}$ and

$$
\widetilde{\mathcal{M}}^{\prime}(\alpha)=\left\{e_{j} M_{\mu}^{\prime}\left(\alpha_{j}\right)\right\}_{j \in \mathbb{Z}} .
$$

This being said we can now prove the following

3. Theorem. Assume the conditions $\mathbf{F 1 )}$ and $\mathbf{F 2}$ ) are satisfied, and that $h \in$ $L^{\infty}\left(\mathbb{R}, L^{2}([0, \pi])\right)$ is 1 -periodic with respect to $t$ and

$$
\left\|\int_{0}^{\pi} h(x, t)^{2} d x\right\|_{\infty}=1
$$

Assume, further, that $\mu_{0} \in \mathbb{R}$ exists such that the function

$$
\bar{M}(\tau):=\delta \int_{-\infty}^{\infty} \dot{\gamma}(t)^{2} d t-\mu_{0} \sqrt{\frac{2}{\pi}} \int_{-\infty}^{\infty} \int_{0}^{\pi} \dot{\gamma}(t)^{2} h(x,(t+\tau) / i) \sin (i x) d x d t
$$

has a simple zero at $\tau=\tau_{0} \in[0,1]$ (that is $\bar{M}\left(\tau_{0}\right)=0$ and $\bar{M}^{\prime}\left(\tau_{0}\right) \neq 0$ ).

Then there exist $\bar{\rho}>0, \bar{\epsilon}>0$ and $\bar{\mu}>0$ such that for any $0<\epsilon<\bar{\epsilon}$, $\left|\mu-\mu_{0}\right| \leq \bar{\mu}$ and $m>\epsilon^{-3 / 4}$, with $m=k i$ and $k \in \mathbb{N}$, there is a continuous function $\alpha: \mathcal{E} \times \mathbb{N}_{m} \times\left(\mu_{0}, \mu_{0}\right) \times(0, \bar{\epsilon}) \rightarrow \ell^{\infty}(\mathbb{R})$ such that $\alpha(E, m, \mu, \epsilon) \in \ell_{E}^{\infty}$ and a continuous map $\Pi: \mathcal{E} \times(0, \bar{\epsilon}) \rightarrow L^{\infty}\left(\mathbb{R}, H_{0}^{2}([0, \pi])\right)$ such that

$$
u_{E}(x, t, \epsilon):=i^{-1} \Pi(E, \epsilon)(x, i \sqrt{\epsilon} t)
$$

is a weak solution of (1) that satisfies

$$
\operatorname{ess}_{\sup }\|\|_{t \in \mathbb{R}}\left\|u_{E}(x, t, \epsilon)-\sqrt{\frac{2}{\pi}} \gamma_{(E, \alpha(E, m, \mu, \epsilon))}(i \sqrt{\epsilon} t) \sin (i x)\right\|_{W} \leq \bar{\rho},
$$

where $\|\cdot\|_{W}$ is the norm in $W=H_{0}^{2}([0, \pi])$.

Moreover, for any fixed $\epsilon$ the map $\Pi: \mathcal{E} \rightarrow \Pi(\mathcal{E})$ is a homeomorphism satisfying

$$
\Pi(\Sigma(E))(x, t)=\Pi(E)\left(x, t+2 m \epsilon^{-1 / 2}\right)
$$

$\Sigma: \mathcal{E} \rightarrow \mathcal{E}$ being the Bernouilli shift.

Proof. The proof is similar to that of Theorem 2 in $[\mathbf{1}]$ thus we only sketch it. As we have observed it is enough to prove that the equation $G((E, \alpha), m, \sqrt{\epsilon} \mu, \epsilon)=0$ has a unique solution $\alpha=\alpha(E, m, \mu, \epsilon)$ in a neighborghood of $\alpha_{0}=\left\{e_{j} \tau_{0}\right\}_{j \in \mathbb{Z}}$. Then setting

$$
\Pi(E, \epsilon)(x, t)=\sqrt{\frac{2}{\pi}}\left[\sum_{l=1}^{i-1} \phi_{l}(t) \sin (l x)+\left[y(t)+\gamma_{\xi}(t)\right] \sin (i x)+\sum_{j>i} z_{j}(t) \sin (j x)\right]
$$

(with $\phi_{l}(t)=\phi_{l}((E, \alpha(E, m, \mu, \epsilon)), \sqrt{\epsilon} \mu, \epsilon, m ; t)$ etc.) we see that

$$
u_{E}(x, t, \epsilon):=i^{-1} \Pi(E, \epsilon)(x, i \sqrt{\epsilon} t)
$$

satisfies equation (1) and it is the unique solution that satisfies (85). Now, we have seen that equation $G((E, \alpha), m, \sqrt{\epsilon} \mu, \epsilon)=0$ is equivalent to $\widetilde{G}((E, \alpha), m, \mu, \epsilon)=0$ 
but, arguing as in $[\mathbf{1}]$, and using $(82),(83),(84)$ we obtain the existence of a unique $\alpha(E, m, \mu, \epsilon)$ with the properties stated in this Theorem. Thus we only have to prove that $u_{E}(x, t, \epsilon)$ satisfies (86) and that $\Pi$ is continuous. The proof of (86) is the same as in [1, Theorem 2] and depends on the uniqueness of $u_{E}(x, t, \epsilon)$. As for the continuity of $\Pi$ we observe that writing as in (87) we have

$$
\sum_{j>i} j^{4}\left\|z_{j}\right\|_{\infty}^{2}<\infty
$$

As in $\left[\mathbf{1}\right.$, Theorem 2] we see that given a sequence $\{E\}_{n \in \mathbb{N}} \in \mathcal{E}$ there exists a subsequence $\{E\}_{n_{k}} \in \mathcal{E}$ such that $z_{j, E_{n_{k}}}(t)$ converges uniformly on any compact interval of $\mathbb{R}$ to a function $z_{j}(t) \in L^{\infty}(\mathbb{R})$. Then, for any $N \in \mathbb{N}$ and $t \in \mathbb{R}$ we have:

$$
\sum_{j=i+1}^{N} j^{4} z_{j}^{2}(t)=\lim _{k \rightarrow \infty} \sum_{j=i+1}^{N} j^{4} z_{j, E_{n_{k}}}^{2}(t) \leq \rho^{2}
$$

and hence $z(x, t)=\sum_{j>i} z_{j}(t) \sin (j x) \in Z_{\rho}$. This being said the proof of the continuity of $\Pi$ goes as in $[\mathbf{1}]$. The proof is complete.

\section{References}

[1] Battelli, F. and FečKan, M. Chaos in the beam equation, J. Differential Equations 209 (2005), 172-227.

[2] Berti, M and Carminati, C. Chaotic dynamics for perturbations of infinite dimensional Hamiltonian systems, Nonlinear Analysis 48 (2002), 481-504.

[3] Chen, G., Hsu, S-B. And Zhou, J. Chaotic vibration of the wave equation with nonlinear feedback boundary control: progress and open questions, in: G. Chen, X. Yu (Eds.), Chaos Control - Theory and Applications, Lecture Notes in Control and Information Sciences (LNCIS) 292, Berlin: Springer 2003, 25-50.

[4] Fiedler, B. and Vanderbauwhede, A. Homoclinic period blow-up in reversible and conservative systems, Z. angew. Math. Phys. (ZAMP) 43 (1992), 292-318.

[5] Holmes, P. and Marsden, J. A partial differential equation with infinitely many periodic orbits: chaotic oscillations of a forced beam, Arch. Rational Mech. Anal.76 (1981), 135-165.

[6] LI, Y. Persistent homoclinic orbits for nonlinear Schrödinger equation under singular perturbation, Dynamics of PDE 1 (2004), 87-123.

[7] Smale horseshoes and symbolic dynamics in perturbed nonlinear Schrödinger equations, Journal of Nonlinear Sciences 9 (1999), 363-415.

[8] Existence of chaos for nonlinear Schrödinger equation under singular perturbation, Dynamics of PDE 1 (2004), 225-237.

[9] Chaos and shadowing lemma for autonomous systems of infinite dimensions, J. Dyn. Diff. Eq. 15 (2003), 699-730.

[10] Homoclinic tubes and chaos in perturbed sine-Gordon equation, Chaos, Solitons and Fractals 20 (2004), 791-798.

[11] Chaos and shadowing around a heteroclinically tubular cycle with an application to sine-Gordon Equation, Studies in Applied Mathematics 116 (2006), 145-171.

[12] Li, Y. And McLaughlin, D. Morse and Melnikov functions for NLS Pde's., Comm. Math. Phys. 162 (1994), 175-214.

[13] Li, Y., Mclaughlin, D., Shatah, J. and Wiggins, S. Persistent Homoclinic Orbits for Perturbed Nonlinear Schrödinger Equation, Comm. Pure and Appl. Math. 49 (1996), 11751255.

[14] Rodrigues, H. M. And Silveira, M. Properties of bounded solutions of linear and nonlinear evolution equations: homoclinics of a beam equation, J. Differential Equations 70 (1987), 403-440.

[15] ZeLiK, S.V. The attractor for a nonlinear reaction-diffusion system in the unbounded domain and Kolmogorov's $\epsilon$-entropy, Math. Nachr. 232 (2001), 129-179. 
[16] The attractor for a nonlinear hyperbolic equation in the unbounded domain, Disc. Cont. Dyn. Sys. Ser. A 7 (2001), 593-641.

[17] - Attractors of reaction-diffusion systems in unbounded domains and their spatial complexity, Comm. Pure Appl. Math. 56 (2003), 584-637.

[18] Zнао, Y. Introduction to some methods of chaos analysis and control for PDEs, in: $G$. Chen, X. Yu (Eds.), Chaos Control - Theory and Applications, Lecture Notes in Control and Information Sciences (LNCIS) 292, Berlin: Springer 2003, 89-115.

[19] YAGasAKI, K. Homoclinic and heteroclinic behaviour in an infinite-degree-of-freedom Hamiltonian system: chaotic free vibrations of an undamped, buckled beam, Phys. Lett. A $\mathbf{2 8 5}$ (2001), 55-62.

Dipartimento di Scienze Matematiche, Università di Ancona, Via Brecce Bianche 1, 60131 ANCONA - ITALY

E-mail address: fbat@dipmat.unian.it

Department of Mathematical Analysis and Numerical Mathematics, Comenius UniVersity, Mlynská dolina, 84248 Bratislava - Slovakia

E-mail address: Michal.Feckan@fmph.uniba.sk

Dipartimento di Scienze Matematiche, Università di Ancona, Via Brecce Bianche 1, 60131 ANCONA - ITALY

E-mail address: franca@dipmat.univpm.it 